\title{
EchoGéo
}

\section{L'environnement socio-spatial comme facteur d'émergence des maladies infectieuses}

Le chikungunya dans l'océan Indien

François Taglioni et Jean-Sébastien Dehecq

\section{OpenEdition}

\section{Journals}

Édition électronique

URL : https://journals.openedition.org/echogeo/11168

DOI : 10.4000/echogeo.11168

ISSN : 1963-1197

Éditeur

Pôle de recherche pour l'organisation et la diffusion de l'information géographique (CNRS UMR 8586)

Référence électronique

François Taglioni et Jean-Sébastien Dehecq, «L'environnement socio-spatial comme facteur d'émergence des maladies infectieuses », EchoGéo [En ligne], 9 | 2009, mis en ligne le 17 juillet 2009, consulté le 31 juillet 2021. URL : http://journals.openedition.org/echogeo/11168 ; DOI : https://doi.org/ 10.4000/echogeo. 11168

Ce document a été généré automatiquement le 31 juillet 2021.

EchoGéo est mis à disposition selon les termes de la licence Creative Commons Attribution - Pas d'Utilisation Commerciale - Pas de Modification 4.0 International (CC BY-NC-ND) 


\title{
L'environnement socio-spatial comme facteur d'émergence des maladies infectieuses
}

\author{
Le chikungunya dans l'océan Indien
}

François Taglioni et Jean-Sébastien Dehecq

\section{Introduction}

1 L'intérêt des recherches en sciences sociales et humaines pour le champ de la santé va crescendo depuis plusieurs années. Il est vrai que les risques sanitaires représentent aujourd'hui des enjeux sociétaux qui se sont multipliés, mondialisés, amplifiés et qui déroutent les sphères décisionnelles de l'espace public, privé ou politique. En matière de sécurité sanitaire, les multiples crises de ces dernières années semblent avoir définitivement consacré la prise de conscience de la nécessaire articulation entre épidémiologie et sciences de l'homme de la société. Si l'étude d'un virus et de son vecteur demeure évidemment prioritaire pour comprendre la dynamique d'une épidémie, les questions demeurées sans réponse lors des récentes crises du chikungunya ou de la dengue révèlent également qu'une part de l'explication de cette dynamique est sans doute à rechercher dans la vaste sphère des comportements humains contextualisés. C'est en effet dans l'infinie variété des usages et des pratiques sociales de l'environnement, dans ce rapport complexe à «l'espace du proche", que toute épidémie trouve des terrains plus ou moins favorables à son développement. $\mathrm{Ce}$ faisant, d'un point de vue méthodologique, on bute rapidement sur la question de la connaissance de ces contextes locaux, de ces environnements proches à la croisée du spatial et du social et qui déterminent autant de territorialités particulières définissant des conditions variables de survenue et de propagation d'une épidémie.

2 Dans un précédent article (Taglioni 2009a) nous nous sommes attachés à étudier les risques et les potentialités de mondialisation du chikungunya et de ses vecteurs ainsi qu'à comprendre comment un problème de pays en développement s'était immiscé 
dans "l'intimité" des pays industrialisés en passant par un premier sas que constituent La Réunion et Mayotte en tant qu'appendices ultrapériphériques de l'Union européenne. Dans la présente étude, nous allons plus directement nous interroger d'une part sur les facteurs d'émergence à l'échelle régionale, celle du sud-ouest de l'océan Indien, et d'autre part, en opérant un changement d'échelle, aux effets de contexte à La Réunion lors de la première vague de l'épidémie de janvier à août 2005. Cette approche multiscalaire, qui met en perspective les points de vue croisés du géographe et de l'épidémiologiste/entomologiste, permet de vérifier l'hypothèse qu'« un phénomène de santé ne s'exprime pas toujours de la meilleure façon à l'échelle la plus fine, des agrégations spatiales raisonnées peuvent être porteuses de sens non fourni par des informations individuelles, ou regroupées à des échelles fines " (Salem, 1998 cité par Vallée, 2008) et vice versa. La réflexion proposée est donc de réfléchir à deux échelles à la complémentarité des effets de contexte socio-spatial et des conditions de développement humain, qui constituent la toile du fond d'une société locale ancrée dans son territoire avec ses usages et ses pratiques. Nous souhaitons ainsi définir et qualifier précisément quelques contextes spécifiques à partir d'un ensemble de variables à l'échelle de la région du sud-ouest de l'océan Indien d'une part et des quartiers de La Réunion d'autre part.

\section{Quelques facteurs d'émergence du chikungunya à l'échelle régionale}

3 Parmi le grand nombre possible d'aléas dans le sud-ouest de l'océan Indien (cyclones, pluies torrentielles, volcanisme, tsunami, maladies infectieuses...) certains sont plus dévastateurs et présents que d'autres. Sur la période récente, l'épidémie de chikungunya, qui s'est déroulée de 2004 à 2006 dans le sud-ouest de l'océan Indien, est sans doute l'événement majeur qui a marqué les hommes, les sociétés et les esprits aussi. Une collecte minutieuse de données permet d'établir une carte (fig. 1, carte commentée dans Taglioni 2009a) dela progression et la prévalence du chikungunya dans la région. Les taux de prévalence ${ }^{1}$ sont dans certains cas obtenus par des enquêtes de séroprévalence (La Réunion, Mayotte, Maurice) et, dans d'autres cas, ils sont estimés (Comores, Seychelles, Lamu). Dans tous les cas ces taux de prévalence nous donnent une image correcte du taux de malades présents dans les différentes populations $d u$ sud-ouest de l'océan Indien par rapport à la population totale durant l'épidémie entre 2004 et 2007 selon les pays. On constate que les taux de prévalence sont variables puisqu'ils s'échelonnent de $25 \%$ aux Seychelles à $75 \%$ à Lamu. Pour essayer de caractériser les contours de la vulnérabilité des Etats et territoires touchés par le chikungunya dans le sud-ouest de l'océan Indien, nous prendrons 5 variables principales, en rapport avec le niveau de développement humain, social et sanitaire, que sont :

- Le niveau général de développement humain

- L'urbanisation et concentration de la population

- Le degré d'ouverture des territoires

- Les flux de personnes

- L'information et la communication

Ces 5 variables sont classiquement retenues pour l'étude des facteurs majeurs d'émergences des maladies infectieuses (Fagherazzi-Pagel, 2007; Raoult, 2005; 
Gonzalez, 2004). Nous allons en vérifier la pertinence à une échelle moyenne, celle du sud-ouest de l'océan Indien.

Figure 1. La progression et la prévalence du chikungunya dans le sud-ouest de l'océan Indien durant l'épidémie de 2004-2007

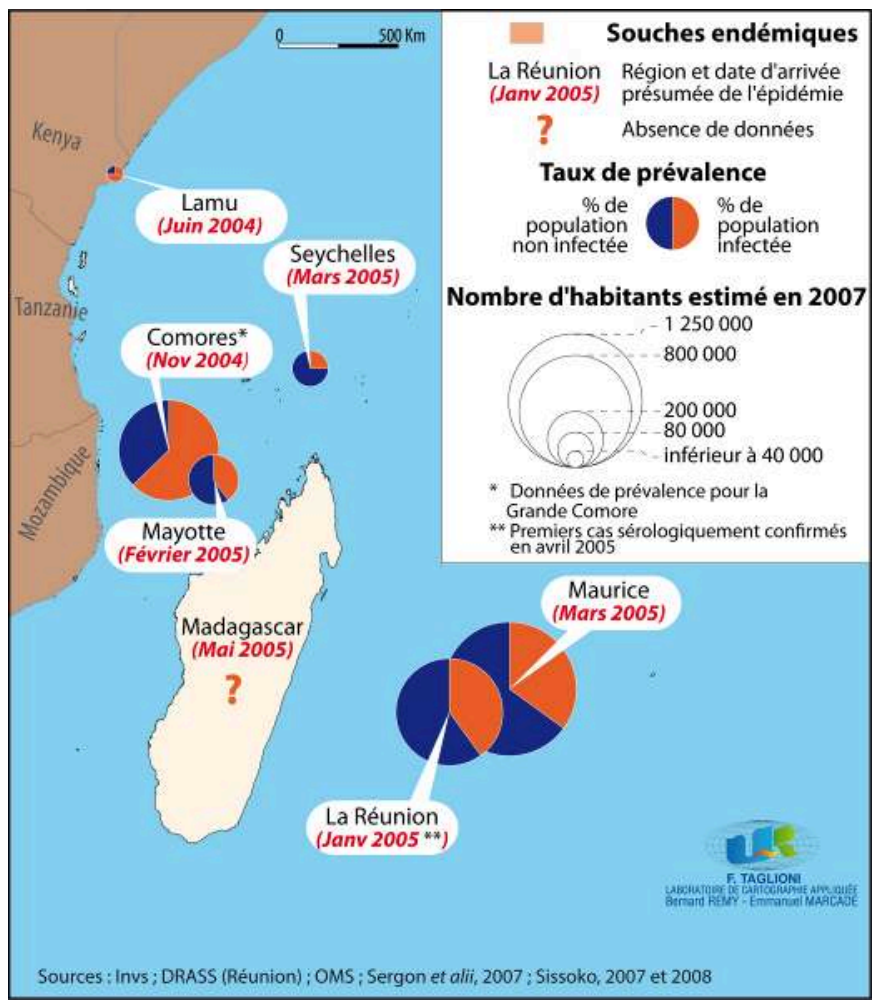

\subsection{Le niveau général de développement humain}

\section{Des situations contrastées de développement humain}

La région du sud-ouest de l'océan Indien est ici définie comme celle composée de La Réunion, Madagascar, Maurice, Mayotte, Seychelles et l'Union des Comores. Pour les besoins spécifiques de l'étude, l'île de Lamu sera intégrée dans certaines analyses dans la mesure où l'épidémie de chikungunya dans le sud-ouest de l'océan Indien est manifestement passée par cet archipel. Cet ensemble géographique s'appréhende bien davantage en termes de diversité que d'unité. On y trouve La Réunion qui est un îlot de prospérité avec cependant des problèmes spécifiques de chômage, de retards sociaux ou encore de santé publique (alcoolisme, diabète). Les situations de Maurice, Mayotte et des Seychelles indiquent un décollage économique, endogène ou exogène et, une transition sanitaire en voie d'achèvement avec néanmoins de l'exclusion et de la pauvreté. D'autres pays sont quant à eux marginalisés économiquement, socialement et politiquement (Madagascar, Union des Comores). Pour préciser les niveaux de développement de ces espaces on peut se référer à l'Indice de développement humain (IDH) tout en gardant à l'esprit que l'IDH (fig. 2) ne prétend nullement être le reflet exhaustif d'un concept aussi complexe que le développement humain. 
6 Si l'on s'en tient aux limites retenues par le Programme des Nations unies pour le développement (PNUD), La Réunion, Maurice et les Seychelles font partie des pays au développement humain élevé avec en premier lieu La Réunion qui présente globalement des normes européennes de développement. Il est certain que son lien institutionnel avec la France et l'Union européenne est garant, pour le moment, de flux de développement essentiels. Cette situation privilégiée de La Réunion se retrouve pour les autres outre-mer français de la Caraibe et du Pacifique en dépit des revendications sociales et identitaires qui ont durement secoué les départements français d'Amérique en février et mars 2009. Si Maurice a réussi son "miracle " économique c'est au détriment de certaines catégories ethno-religieuses issues du communalisme. D'autre part, au sein de la République mauricienne, l'île de Rodrigues souffre de son relatif enclavement et de son statut d'île secondaire (Taglioni 2005). Depuis les débuts de la colonisation au XVI ${ }^{\mathrm{e}}$ siècle, et après l'indépendance en 1968, Rodrigues était considérée comme une simple dépendance assez lointaine $(700 \mathrm{~km})$ de l'île Maurice. Au terme de près d'un siècle de combats des Rodriguais, l'autonomie s'est concrétisée en 2001. En dépit de cette autonomie le tremplin vers un meilleur développement économique et social se fait toujours attendre. Aux Seychelles, petit Etat peuplé de 80000 habitants, la prospérité économique se fonde fortement sur les services en général et le tourisme en particulier avec les risques inhérents d'une économie aussi spécialisée.

7 Un deuxième groupe constitué de Madagascar, Maldives, Mayotte, Rodrigues et Union des Comores se retrouve derrière l'étiquette de pays au développement moyen. Dans les faits, Mayotte, outre-mer français connaît une embellie de son niveau de vie et de ses infrastructures de développement. La collectivité départementale n'a pas encore atteint les standards de sa voisine, La Réunion, qui jouit d'un statut de département, ni de celui de la métropole, mais elle est néanmoins installée dans une spirale de développement avec cependant quelques limites (Taglioni 2008). Le développement humain dans l'Union des Comores est très fragile avec par exemple un fort taux de mortalité infantile, un niveau d'alphabétisation inférieur à $60 \%$ et une espérance de vie assez limitée pour ne prendre que quelques indicateurs. Ces résultats traduisent que près de $50 \%$ de la population vit sous le seuil de pauvreté. L'instabilité politique qui règne sur l'Union est une des clés de ce mal développement. A Madagascar, la situation fluctue au gré des crises politiques et elle reste préoccupante car la majorité de la population vit sous le seuil de pauvreté, notamment en zone rurale. La Banque mondiale classe d'ailleurs plus sévèrement Madagascar que le PNUD puisqu'elle range, à juste titre nous semble t-il, ce pays dans le groupe des moins avancés (PMA). L'archipel de Lamu, en dépit d'une réputation de paradis tropical, doit faire face à la dure réalité d'îles ou les incertitudes de développement sont réelles. Les indicateurs classiques $\mathrm{du}$ développement sont tous dans le rouge (espérance de vie, analphabétisme, mortalité infantile ...).

8 A la lumière de cette analyse, on ne peut pas dire que l'IDH soit hautement significatif en tant qu'indicateur d'émergence du chikungunya pour les pays au développement élevé comme La Réunion, Maurice ou les Seychelles. En effet, en dépit de leur bon niveau général de développement ces espaces sont touchés à plus de $25 \%$ de leur population par l'épidémie, c'est d'ailleurs cela même qui a surpris dans le cas de La Réunion dont le taux est supérieur à celui des Seychelles ${ }^{2}$ qui est pourtant globalement en dessous des standards de développement du département français. Il y a donc d'autres facteurs explicatifs et nous y reviendrons. En revanche, pour les pays au 
développement humain moyen voire faible comme l'Union des Comores ou Lamu les taux de prévalence sont plus élevés et indiquent que des conditions précaires de développement humain vont de pair avec une flambée épidémique importante (supérieure à $50 \%$ ). Pour Mayotte la réalité est biaisée par son fort taux de population migrante, issue d'une immigration récente et illégale, qui ne connaît pas les mêmes conditions de vie que le reste de la population mahoraise.

Figure 2. L'indice de développement humain dans le sud-ouest de l'océan indien

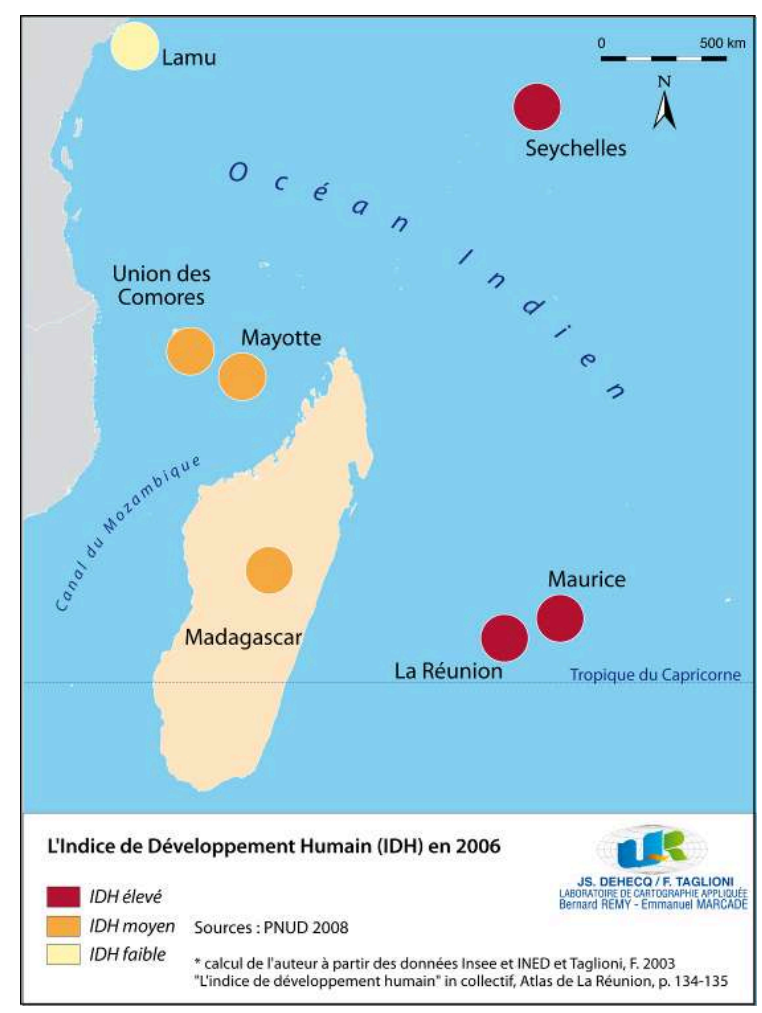

\section{Niveau d'alphabétisation}

9 Bien que dans le calcul de l'IDH entre en considération le niveau d'éducation (taux d'alphabétisation et taux brut de scolarisation), nous allons néanmoins revenir sur la corrélation entre le taux d'alphabétisation et le risque d'émergence. De fait, le niveau d'alphabétisation, pour peu qu'il soit fiable, ce qui n'est pas une certitude dans les pays les moins avancés, pourrait être un bon indicateur de risque d'émergence de maladie infectieuse à transmission vectorielle. Il est en effet prévisible que les comportements les plus favorables des populations vis-à-vis de la lutte antivectorielle (LAV) ou encore de la bonne réception des messages des services publics en matière d'hygiène (utilisation de répulsifs, de moustiquaires imprégnées d'insecticide ...) et de la bonne gestion de son environnement proche (jardin, maison, etc.) sont ceux des populations les plus réceptrices à ces messages et donc a priori celles pour le moins correctement alphabétisées.

Pour le chikungunya, le taux d'alphabétisation (fig. 3) a manifestement une bonne corrélation avec la fréquence de l'infection puisque les populations les moins bien alphabétisées comme celles de Lamu, et des Comores sont celles qui ont été les plus 
massivement touchées par l'épidémie. A l'inverse, les populations de La Réunion, Maurice et les Seychelles qui bénéficient d'un bon système d'éducation et d'alphabétisation ont des prévalences moins élevées. Pour Mayotte il est bien difficile de connaître le taux d'alphabétisation en raison une fois encore du fort taux d'immigrés.

Figure 3. Le taux d'alphabétisation des adultes dans le sud-ouest de l'océan indien

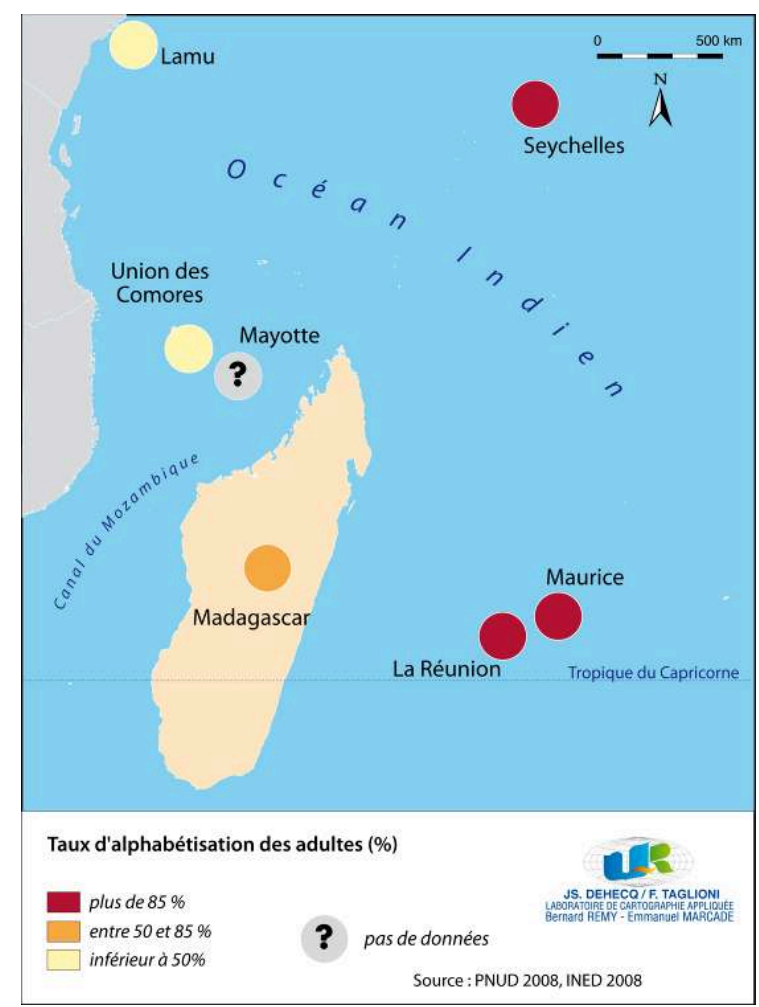

\subsection{Urbanisation et concentration de la population}

11 "Les maladies infectieuses peuvent émerger dans les zones rurales, mais les zones urbaines sont cruciales pour leur dissémination et leur transformation en épidémie, voire en pandémie"3 (Heymann, 2009). De fait, l'urbanisation croissante et la concentration toujours plus forte des populations sont des facteurs d'émergence bien établis des maladies virales à transmission par aérosols qu'encouragent et accentuent la promiscuité et la multiplication des contacts interhumains. Dans le cas du virus chikungunya, à transmission pourtant vectorielle, le niveau d'urbanisation joue aussi un rôle important dans la mesure où Aedes albopictus est un moustique particulièrement bien adapté en milieu urbain et périurbain. Ce moustique est invasif et on le retrouve en position dominante à La Réunion, Maurice, Seychelles (Delatte, 2008a et 2008b ; Mouchet, 1994 ; Mouchet, 1995). Aedes aegypti, autre porteur du chikungunya, est en net recul sur ces espaces, mais il continue d'être majoritaire à Lamu, en Grande Comore et Rodrigues (Charell, 2007 ; Zeller, 1998). A Mayotte les deux Aedes sont à répartition à peu près équivalente avec albopictus qui progresse depuis le début des années 2000 qui est la date de son introduction (Bagny 2009). 

observe que La Réunion, Maurice et Seychelles, avec un Aedes albopictus dominant, sont des îles où l'urbanisation (fig. 4) est très présente avec des taux supérieurs à $60 \%$ sauf à Maurice. Pour cette dernière, les statistiques sont trompeuses à l'épreuve du terrain car dans les faits l'île est marquée par l'urbanisation ou la périurbanisation avec des concentrations importantes dans ces espaces. A Lamu et Grande Comore les taux d'urbanisation sont beaucoup moins élevés, inférieurs à $40 \%$, mais la prévalence de cas de chikungunya est importante avec un Aedes aegypti qui est toujours le plus présent et le mieux adapté à ces espaces ruraux. A Mayotte enfin, l'urbanisation gagne du terrain et Aedes aegypti perd de son emprise face à Aedes albopictus mieux adapté à cette nouvelle donne d'augmentation et de concentration de la population (Bagny, 2009).

Le niveau d'urbanisation est donc un facteur d'émergence significatif dans un contexte mondial du développement conjoint d'Aedes albopictus et de l'urbanisation dans les pays les plus développés bien sûr mais aussi dans les pays au développement retardé. Il faut néanmoins préciser que cette adaptation particulière d'Aedes albopictus est pour le moment très spécifique au sud-ouest de l'océan Indien. Ailleurs, c'est généralement le contraire avec un Aedes albopictus plutôt rural et un Aedes aegypti plutôt urbain. Les entomologistes n'ont pas d'explication à ce jour sur cette spécificité (Bagny, 2009).

Figure 4. Le taux d'urbanisation dans le sud-ouest de l'océan indien

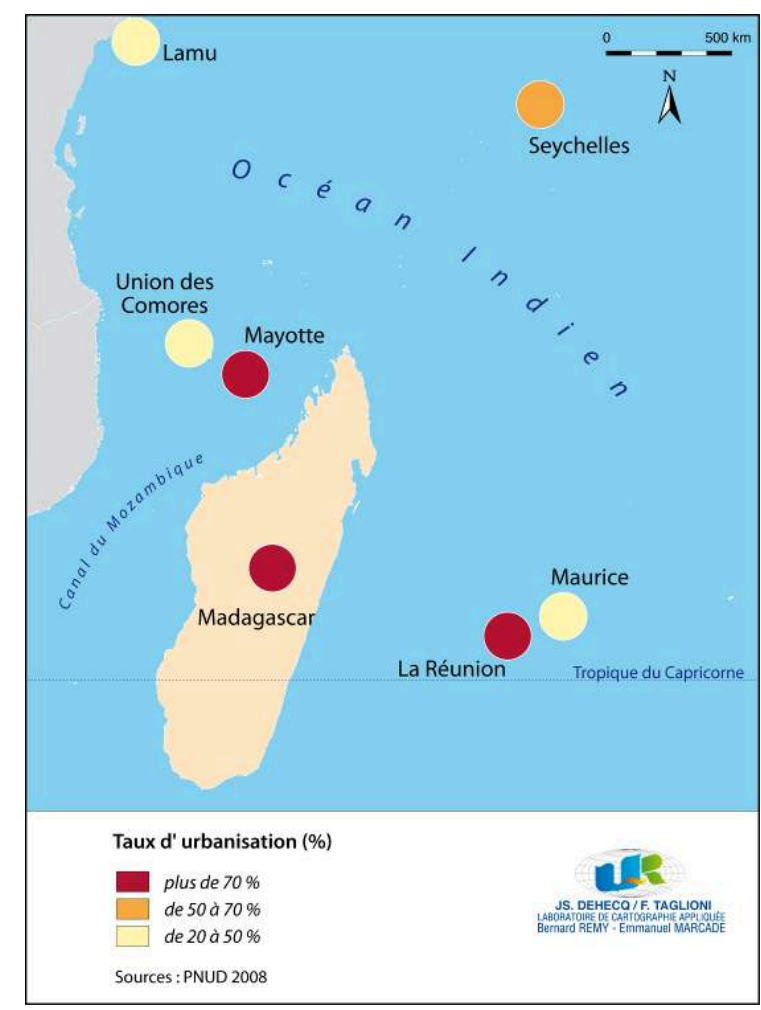

\subsection{Le degré d'ouverture des territoires}

Les Etats et territoires du sud-ouest de l'océan Indien de cette étude sont tous insulaires. On peut faire l'hypothèse que le degré d'ouverture vers l'extérieur sera un 
élément déterminant de la propagation de l'épidémie non seulement à l'échelle régionale mais aussi et surtout à l'échelle mondiale. On a coutume de dire que la fragmentation géographique, générée par la mer et les océans, dans les îles et les archipels est une entrave à la diffusion de l'éducation, des soins de santé, de l'approvisionnement alimentaire, de la technologie, des échanges de biens et de personnes, mais aussi de l'information. Elle serait donc génératrice de discontinuité voire d'isolement. Tout cela est néanmoins à nuancer car l'isolement est aujourd'hui un épiphénomène à la surface du globe et rares sont les lieux ou espaces qui souffrent d'isolement géographique absolu. Les îles n'échappent pas à ce constat et il est vrai que des barrières géographiques fortes (cours d'eau, chaînes de montagnes, déserts, zones glaciales...) peuvent avoir des effets d'isolement et de discontinuité spatiale beaucoup plus nets que la mer. Ainsi, certaines zones continentales du Canada, du Brésil, d'Argentine, d'Amazonie ou des plaines d'Asie centrale sont infiniment plus isolées que les îles du sud-ouest de l'océan Indien. "La barrière géographique que représente la côte de l'île n'est donc finalement que l'un des nombreux mécanismes possibles d'isolement" (Benoist1987). Il faut la situer à sa juste place dans un ensemble de facteurs concourant à l'isolement. Il est sans doute plus juste d'envisager aujourd'hui l'isolement en termes de degrés d'enclavement. La notion d'enclavement nous permet d'abolir la distance linéaire, qui serait l'éloignement, et de la remplacer avantageusement par les notions relatives de distance-temps pour ce qui concerne plus particulièrement le transport des individus par avion et de distance-coût pour ce qui est du transport maritime des marchandises. Ces deux indicateurs, distance-temps et distance-coût, permettent de mesurer de façon plus objective l'accessibilité humaine et commerciale des îles. Ils sont aussi des indicateurs fondamentaux pour apprécier l'insularité qui est, ou a été, intimement liée à l'accessibilité.

15 Ainsi, les Seychelles, les Comores et Maurice, Etats multi-insulaires, présentent des îles capitales (Grande Comore, Maurice, Mahé) qui sont bien ouvertes au monde avec des aéroports internationaux et des compagnies aériennes internationales. En revanche les îles secondaires (Rodrigues, Agalega, Cargados, Saint Brandon, Aldabra, Anjouan, Mohéli) sont moins accessibles et parfois enclavées. Pour La Réunion et Madagascar, l'accessibilité internationale des lieux est très bonne. Pour Mayotte, il n'existe toujours pas de desserte aérienne directe avec la France et les passagers doivent transiter par La Réunion ce qui allonge de façon notable la durée du voyage.

Que ce soit à l'échelle mondiale comme à l'échelle régionale, la propagation de l'épidémie de chikungunya s'est effectuée de deux façons distinctes, soit par les flux de populations migrantes, soit par les touristes internationaux.

\subsection{Flux de personnes : migrations et tourisme}

17 A l'échelle régionale tout d'abord, le cheminement de l'épidémie met en jeu, dans un premier temps, des flux humains entre la côte est de l'Afrique, et plus particulièrement le Kenya, et l'Union des Comores. Ces flux traditionnels et anciens entre les Comores et les îles des archipels de Zanzibar et Lamu ont permis au virus du chikungunya de progresser de Lamu vers la Grande Comore. L'archipel des Comores semble bien avoir été par la suite la "plaque tournante" de l'épidémie qui s'est ensuite propagée à Mayotte, pour des raisons évidentes de flux migratoires de premier ordre entre l'Union des Comores et la collectivité départementale (fig. 5, carte commentée dans Taglioni 
2008), et ensuite à Madagascar (dont les données épidémiologiques sont des plus floues), à La Réunion en raison là aussi de flux migratoires et touristiques soutenus et ensuite vers Maurice et les Seychelles sans certitude sur de la provenance des premiers cas. Ce schéma, bien qu'incomplet faute de données, de la propagation de l'épidémie repose sur des relations intenses de flux humains entre les îles de la région. Cette réticulation des échanges humains n'a rien d'étonnant tant les liens et les réseaux culturels, politiques et économiques sont anciens. L'inconvénient de cette proximité se situe, dans ce contexte, dans la dissémination des maladies infectieuses et notamment de la fièvre de la vallée du rift qui, partie du Kenya, s'est propagée à l'ensemble de l'Afrique subsaharienne puis des Comores, Mayotte (depuis 2007) et Madagascar en suivant des cheminements proches de ceux du chikungunya (Sissoko, 2009 ; Breiman, 2008 ; Anyamba, 2009, Zeller, 1998). De la même manière, le choléra, venu d'Afrique de l'est et du sud, est passé par les Comores et Mayotte (Brettes, 2001). La dengue sévit aussi de façon endémique avec des poussées épidémiques (Sissoko, 2008 ; Ratsitorahina, 2008 ; Zeller, 1998 ; Schwartz, 1994). A ce propos, celle de 1977-78 à La Réunion est tout à fait comparable par son intensité à celle de chikungunya de 2005-2006 (Coulanges, 1979; Metselaar, 1980, Kles, 1994). La Réunion avait enregistré à l'époque une prévalence de l'ordre de 30 à $35 \%$ de la population. La précédente épidémie de dengue remontait à 1873 en provenance de Zanzibar (Aubry, 2008). D'une façon générale, le sud-ouest de l'océan Indien est à la périphérie de l'Afrique du sud et de l'est où circulent sur le mode endémique ou épidémique diverses maladies infectieuses et notamment à transmission vectorielle.

Figure 5. Les flux migratoires de l'Union des Comores vers Mayotte

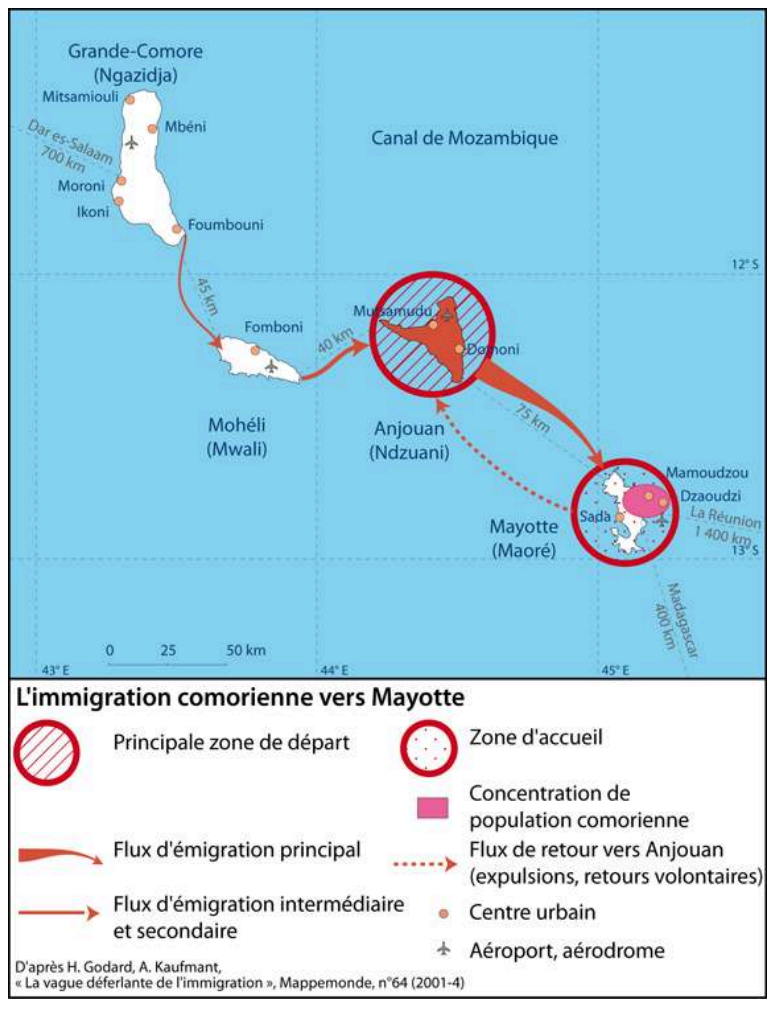

18 A l'échelle mondiale maintenant, les flux touristiques vers le sud-ouest de l'océan Indien (Taglioni 2009b) sont des facteurs d'explication de la diffusion de cas de chikungunya non autochtones en Europe et dans la Caraïbe entre 2005 et 2006. En 
valeur absolue (fig. 6), les destinations les plus fréquentées en 2007 sont Maurice et les Maldives avec respectivement plus de 900000 et près de 700000 touristes. Vient ensuite un groupe intermédiaire constitué de La Réunion et de Madagascar avec un peu moins de 400000 touristes. Les Seychelles et Zanzibar connaissent une fréquentation plus faible avec environ 150000 touristes et enfin Mayotte, les Comores et Rodrigues sont les destinations les moins visitées avec moins de 40000 touristes annuels. Les cas, "importés" par des touristes de retour de la région, de l'ordre de 900 en France (Invs, 2007), d'une centaine au Royaume-Uni, d'une dizaine en Suisse, Italie, Allemagne, Belgique et de quelques cas en Norvège, République Tchèque ou encore dans les départements français d'Amérique, traduisent bien la géographie du tourisme dans le sud-ouest de l'océan Indien. En effet, la provenance des quelques 2,7 millions de touristes en 2007 est très largement européenne (fig. 6). Les Européens sont les "piliers" du tourisme à La Réunion ( $80 \%$ en 2007 dont $75 \%$ de français métropolitains), à Mayotte ( $90 \%$ en 2007 dont $44 \%$ de français métropolitains et $43 \%$ de Réunionnais), à Madagascar (60\%) et à Maurice (67\%). Pour les Seychelles et les Maldives, les trois quarts des visiteurs sont européens, mais la part des Français est moindre alors que les Allemands, les Italiens et les Britanniques sont très présents. Dans l'Union des Comores, les français assurent la moitié des arrivées mais l'autre moitié est constituée des pays de la zone avec l'Afrique Australe et La Réunion en tête. À Rodrigues la situation est particulière puisque ce sont respectivement l'île Maurice et La Réunion qui fournissent le contingent principal de visiteurs. C'est d'ailleurs une des particularités de la région d'entretenir des flux touristiques à faible distance. Dans ces conditions, il n'est pas surprenant que le virus ait circulé entre l'océan Indien et l'Europe avec d'ailleurs une parfaite similitude de la distribution des cas dans le temps notamment entre La Réunion et La France.

Figure 6. Les flux touristiques et la part des Européens dans le sud-ouest de l'océan indien

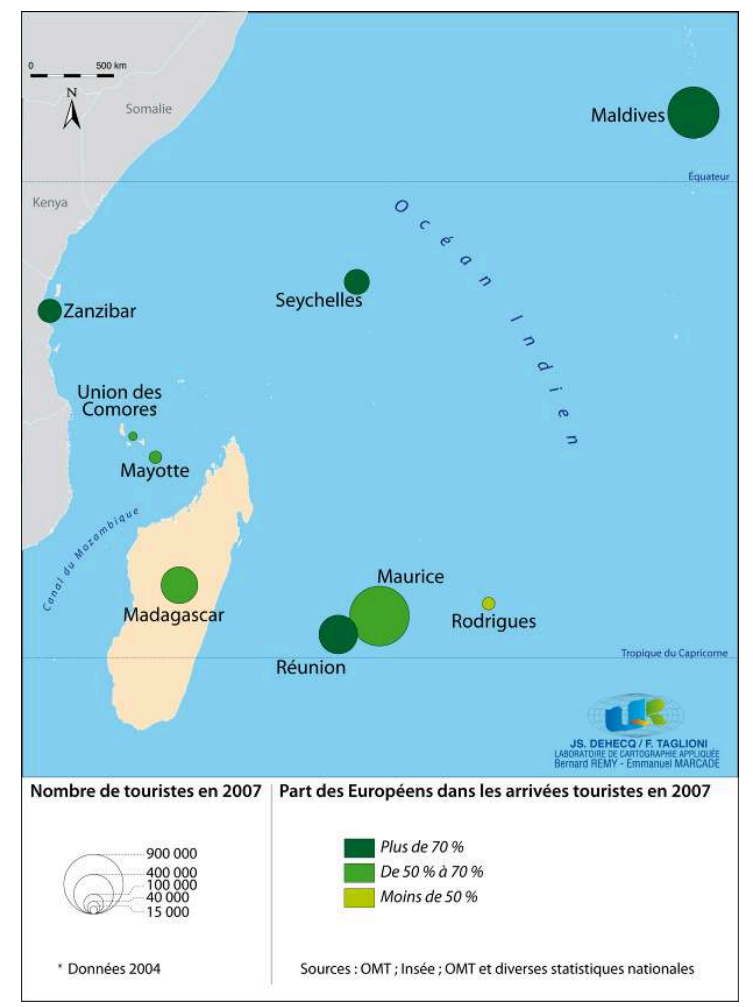


19 A ces cas d'importation, qui constituent une possible étape vers la propagation du chikungunya vers l'Europe et au-delà dans la mesure où le vecteur est parfois présent dans ces pays, s'ajoute la première épidémie autochtone au monde hors d'un pays tropical. Il s'agit de celle qu'a connu l'Italie entre juillet et septembre 2007 où 166 cas, sur plus de 200 cas suspects, ont été confirmés en Emilie-Romagne (Mir, 2008; Rezza, 2007). Il semble que la maladie se soit propagée à partir d'un voyageur de retour d'Inde et porteur du virus. C'est en tout cas la preuve que cette maladie peut s'installer hors de la zone intertropicale via les flux de touristes internationaux si un vecteur potentiel est présent.

\subsection{Information et communication : une épidémie abordée différemment}

Information et communication sont des éléments déterminants dans les campagnes de prévention des maladies infectieuses mais ils le sont aussi lorsque les épidémies sont déclarées. Sans être à proprement parler des facteurs d'émergence de la maladie, information et communication sont en revanche des rouages fondamentaux du mécanisme de déclenchement de crise sanitaire et d'autre part des éléments forts d'une éventuelle propagation de l'épidémie hors des frontières des foyers épidémiologique de départ.

21 La façon dont est analysée et traitée l'épidémie du chikungunya est différente selon les gouvernements concernés, le traitement médiatique et de la vitalité de la société civile dans l'espace public des différents pays affectés. Pour le seul sud-ouest de l'Océan Indien, les situations sont de facto très contrastées. La Réunion présente clairement une "surinformation » sur la situation sanitaire et une appropriation totale de la crise par l'espace public médiatique (Idelson 2009 et Watin 2009). Cette situation a généré un courant de rumeurs et de polémiques qui a contribué à troubler les messages de prévention officiels, à entretenir la tension médiatique et à mobiliser de façon exceptionnelle l'opinion publique dans l'île et au-delà. La situation de l'information et de la communication est du reste paradoxale sur les presque deux ans que dure l'épidémie. Durant la première phase de l'épidémie en 2005, qui a vu plusieurs milliers de cas avec un pic de près de 450 malades la semaine du 9 au 15 mai, les médias sont discrets, la maladie est réputée bénigne, l'opinion publique n'est pas en alerte. On parle d'une maladie proche de la dengue avec de la fièvre et des douleurs articulaires. Les autorités sanitaires et publiques rassurent et banalisent les faits. Lors de cette première épidémie de 2005, aucun certificat de décès mettant en cause de façon directe ou indirecte n'est mentionné. La fin de l'année 2005 et surtout le début de l'année 2006 sont d'une toute autre couleur médiatique et épidémiologique. Les chiffres s'affolent, 10000 cas de chikungunya dès la deuxième semaine de 2006, plus de 25000 la quatrième semaine, paroxysme la semaine suivante avec plus de 45000 cas en 7 jours! Et cela continue les semaines suivantes avec une décrue mais tout de même près de 5 000 cas encore la première semaine d'avril 2006. Les certificats de décès s'accumulent avec un total final de plus de 250, des symptômes qui dépassent en intensité et en durée ceux que décrivaient la médecine et les médias. C'est donc la crise sanitaire totale à La Réunion puissamment relayée et mis en scène (fig. 7) par les médias réunionnais et métropolitains. En ce qui concerne la presse écrite, les trois quotidiens de l'île, Le journal de l'île de La Réunion, Le quotidien et Témoignages, consacrent plus de 9000 
articles entre 2005 et 2007 ! La presse nationale française cumule plusieurs centaines d'articles. Le Figaro, Le Monde, Libération, L'Humanité et La Croix sont les quotidiens les plus mobilisés par l'épidémie.

La «surinformation » quantitative, que l'on vient de décrire succinctement, n'est pas obligatoirement synonyme d'information objective. Ainsi, La République de Maurice, en dépit d'une presse écrite qui a produit plusieurs centaines d'articles dans ses deux principaux quotidiens, L'Express et le Mauricien, se caractérise surtout par une "sousinformation» destinée à minimiser l'impact de l'épidémie pour préserver l'économie en général et le tourisme en particulier (cf. Taglioni 2009b). Les autorités sanitaires mauriciennes ont pendant longtemps annoncé un chiffre de quelques milliers de cas de chikungunya avant que des études mettent en évidence plus de 400 000 malades et une surmortalité importante en 2006 (Beesoon 2008). Il n'y a donc pas eu de tension médiatique. En effet, le rôle des médias était surtout de rassurer les ressortissants nationaux et les touristes internationaux. Néanmoins, cette information quelque peu déconnectée des réalités de terrain n'a heureusement pas eu, comme on aurait pu le craindre, d'impact sur le nombre de touristes qui est rentré malade de Maurice car les autorités sanitaires ont multiplié les efforts de lutte antivectorielle dans les hôtels et leurs abords. Elles ont par ailleurs encouragé les visiteurs à se protéger contre les piqures et à limiter leurs déplacements sur l'île. Pour ce dernier point, la forme du tourisme balnéaire à Maurice, dans des hôtels et des complexes hôteliers prévus pour faire du tourisme captif, a facilité les choses.

Figure 7. Première de couverture du JIR du 22 avril 2006

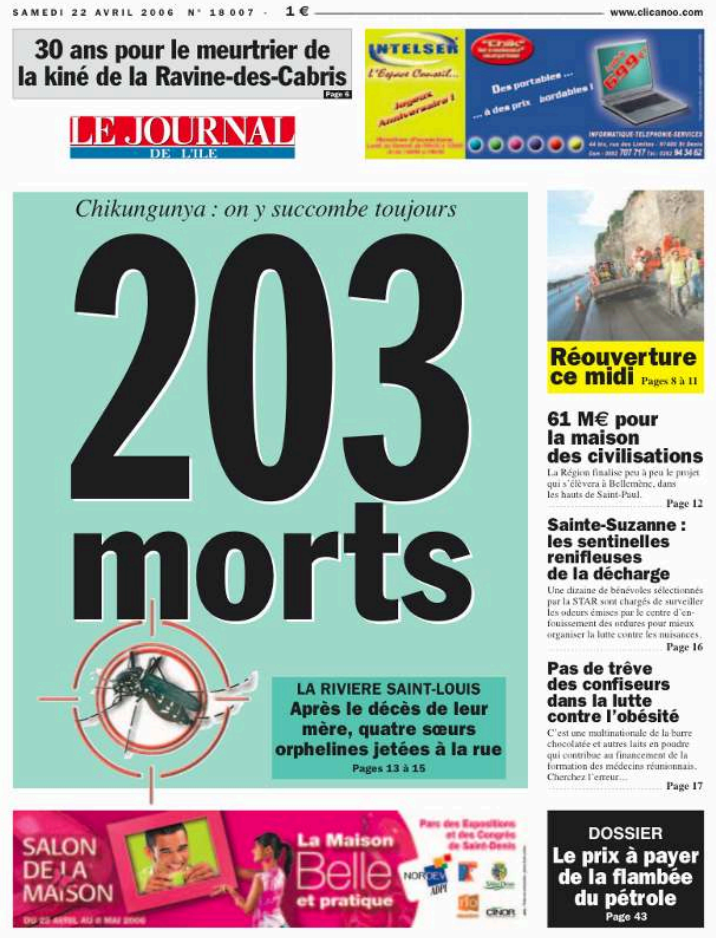

La situation de Mayotte est bien distincte de celle de La Réunion. Ces deux outre-mers français présentent en effet un héritage historique, un contexte culturel et un statut politico-institutionnel très différents. En ce qui concerne plus spécifiquement la presse 
écrite, un certain nombre de paramètres sont essentiels pour expliquer que l'épidémie de chikungunya n'a pas été vécue comme une crise médiatique ou épidémique. Il faut d'abord considérer qu'il n'existe pas de presse écrite quotidienne à Mayotte mais une presse hebdomadaire, comme Le Mahorais, Mayotte hebdo ou encore Mawana, qui est complétée par une presse sur Internet. Ces médias francophones sont assez peu fréquentés car d'une part la population mahoraise ne présente pas plus de $50 \%$ de francophones réels (sans compter qu'environ un quart de la population est constituée de clandestins comoriens non ou peu francophones) et que d'autre part, l'illettrisme est élevé à Mayotte. Enfin, le faible pouvoir d'achat moyen à Mayotte est un frein certain à la diffusion de la presse payante que ce soit sous forme papier en encore plus sous forme électronique sur Internet car cela suppose un équipement informatique et une connexion haut débit. Le faible pouvoir d'achat est aussi un frein aux consultations médicales aujourd'hui payantes pour les mahorais et très risquée pour les clandestins en situation irrégulière. Le recours à la médecine traditionnelle reste encore pratique courante et minimise ainsi la pression sur les médecins et les hôpitaux en période épidémique. Comme à Maurice mais pour des raisons très différentes, il n'y a finalement pas eu de crise sanitaire à Mayotte, pas plus qu'une polémique sur le nombre de cas, en dépit au final d'une prévalence très proche de celle de La Réunion ou de Maurice.

Dans l'Union des Comores la situation est en certains points comparable à celle de Mayotte dans la mesure où les médias sont peu présents dans la sphère publique en raison de leur coût, de l'illettrisme et de la confidentialité de réseaux Internet. Les autorités comoriennes, qui sont montrées du doigt comme étant à l'origine de l'épidémie, ont essayé de présenter une bonne transparence dans les statistiques épidémiologiques et ses conséquences socio-économiques pour le pays. Le gouvernement comorien affirme avoir prévenu les autorités réunionnaises de l'épidémie de chikungunya qui touchait Grande Comore (prévalence supérieure à $60 \%$ ) mais sans réponse. Cela confirmerait la difficulté d'une coopération sanitaire régionale et plus encore internationale. Par delà les graves problèmes de santé publique dans l'Union des Comores, la plus grande menace qui pèse sur l'archipel est celui d'une crise institutionnelle bien plus que sanitaire. L'objectif premier du gouvernement est donc de préserver l'unité politique très fragile de la fédération (Taglioni 2008).

Madagascar présente une information assez partielle et limitée. Les trois principaux quotidiens, L'express de Madagascar, La tribune et Midi Madagascar n'ont guère publié que moins de 100 articles durant l'épidémie et le plus souvent en se faisant l'écho de la situation épidémiologique à La Réunion ... mais en occultant beaucoup la situation nationale. Avec le recul, on ne peut que douter d'une déclaration dans la presse du ministre de la santé de Madagascar qui en mars 2006, au plus fort sans doute de l'épidémie, reconnaît des cas sporadiques après la visite du directeur général de l'Organisation mondiale de la santé (OMS). La presse malgache se fait l'écho de cette déclaration et insiste davantage sur l'épidémie de dengue, qui est en fait endémique à Madagascar; elle inquiète moins les touristes et les populations car mieux connue de tous mais pourtant tout aussi virulente, et dans certains cas mortelle ${ }^{4}$, que le chikungunya.

La situation des Seychelles est à mi-chemin entre celle de Maurice et de La Réunion. D'un coté l'épidémie a été minimisée pour préserver le tourisme déjà mal en point. Le site Internet d'Air Seychelles ${ }^{5}$ est édifiant à ce propos car il annonce, dans son édito du 
28 mars 2006, que "L'éradication du virus est en passe d'être atteinte". Dans les faits l'épidémie se poursuit et connaîtra même un deuxième pic fin mai 2007 alors qu'à cette date La Réunion et Maurice sont entrées en phase inter-épidémique et qu'elles ne connaissent plus que quelques cas sporadiques. D'un autre côté, la presse seychelloise fait un large travail d'information diffusé notamment par le quotidien Seychelles nation.

A l'échelle du sud-ouest de l'océan Indien, et sans vouloir généraliser à tout prix, certaines variables influent sur le niveau de vulnérabilité des populations face au chikungunya comme on vient de le montrer. Néanmoins, à aléa égal le risque n'est pas proportionnellement plus important dans les pays réputés les plus vulnérables. Ce constat est d'autant plus troublant que l'on aurait pu imaginer que les conditions socioéconomiques et sanitaires à La Réunion, département français et région européenne qui bénéficie de subventions importantes pour son développement, sont telles qu'une crise sanitaire de ce type ne pouvait pas se produire car la vulnérabilité semblait moindre que dans des pays plus pauvres de la région. Les progrès sanitaires et sociaux des cinquante dernières années, avec notamment une baisse continue et spectaculaire de la mortalité infantile ainsi qu'un allongement important de l'espérance de vie, ont conduit à un relâchement de la prévention en matière de lutte antivectorielle dont le service est passé de plus de 160 personnes dans les années 80 à moins de 20 personnes au début de l'épidémie en 2005. Il est vrai que l'éradication du paludisme à la fin des années 80 avait conforté l'idée des autorités sanitaires que la menace d'épidémie virale par transmission d'arthropodes était révolue. C'était une erreur... comme nous allons le voir en détail en se plaçant maintenant à l'échelle d'un territoire, celui de La Réunion, pour lequel nous avons des données précises et fiables sur l'épidémie de chikungunya. L'analyse spatiale de l'épidémie va faire apparaître des effets de contextes plus spécifiques, sans être d'ailleurs forcément plus significatifs, qu'à l'échelle régionale.

\section{Les effets de contexte à La Réunion}

Au-delà de la densité vectorielle, les facteurs de l'environnement socio-spatial jouent un rôle important dans la propagation et l'ampleur d'une épidémie en conditionnant le risque d'exposition d'une population à un agent pathogène (Harpham, 1991; Knudsen, 1992). L'écologie urbaine et les comportements des populations sont les principaux composants à étudier afin de caractériser l'exposition des populations (Ruiz, 2004 ; Thammapalo, 2005). Toutes les études menées dans ce sens ne démontrent pas systématiquement l'impact de ces facteurs environnementaux, mais elles mettent en avant la complexité des interactions entre variables, la difficulté de définition d'une échelle géographique pertinente pour l'analyse des données et la variabilité des comportements individuels à risque (Younus, 2007 ; Onwujeke, 2005).

L'objectif de cette partie est donc la caractérisation socio-spatiale des foyers primaires de chikungunya à La Réunion lors du premier pic épidémique d'avril à juin 2005. Ceci afin de comprendre pourquoi l'épidémie a commencé dans ces quartiers et définir les facteurs permettant d'éditer la carte des quartiers à risques de La Réunion en fonction des effets de contexte. La période d'étude retenue s'étend de janvier à août 2005, mois où toutes les enquêtes réalisées par le service de lutte antivectorielle de la Direction régionale des affaires sanitaires et sociales (DRASS) suite aux signalements par la Cellule Inter Régionale d'Epidémiologie (CIRE) (Paquet, 2006) ont été géoréférencées. Ce géoréférencement de près de 3000 cas est assez exceptionnel par son nombre et pour 
une épidémie d'une telle importance (prévalence de $38 \%$ et environ 300000 cas sur les 800000 habitants que compte La Réunion) permet des analyses spatio-temporelles fines de cette phase épidémique.

\subsection{Le cadre de l'étude}

Un indicateur du risque d'introduction du virus du chikungunya à La Réunion pourrait se fonder sur l'origine des cas importés de paludisme sur l'île. En effet, de façon récurrente, on observe durant la période des fêtes de fin d'année et de congés d'été austral une recrudescence des cas importés de paludisme à La Réunion. Ils se superposent avec la période de retour de congés annuels. Les Comores et Madagascar sont les 2 principaux pays d'origine de ces cas (Sissoko, 2006). La répartition spatiale, $a$ priori, aléatoire de ces cas sur l'île (fig. 8), avec 11 communes sur 24 présentant des cas importés de paludisme entre octobre 2004 et mars 2005, révèle bien cette dispersion sur tout le territoire et démontre que ces personnes, si elles étaient porteuses du virus du chikungunya, auraient pu être à l'origine de foyers dans de nombreuses communes de l'île. Cette référence au paludisme, éradiqué depuis 1979 à La Réunion, permet d'affirmer que le virus du chikunguya a été introduit en de nombreux endroits de l'île avec néanmoins un tropisme avéré par l'apparition des premiers foyers de transmission. C'est sur ces premiers foyers et la recherche des facteurs de leur émergence que porte cette partie de l'article.

Figure 8. Distribution des 74 cas de paludisme entre le dernier trimestre 2004 et le premier trimestre 2005

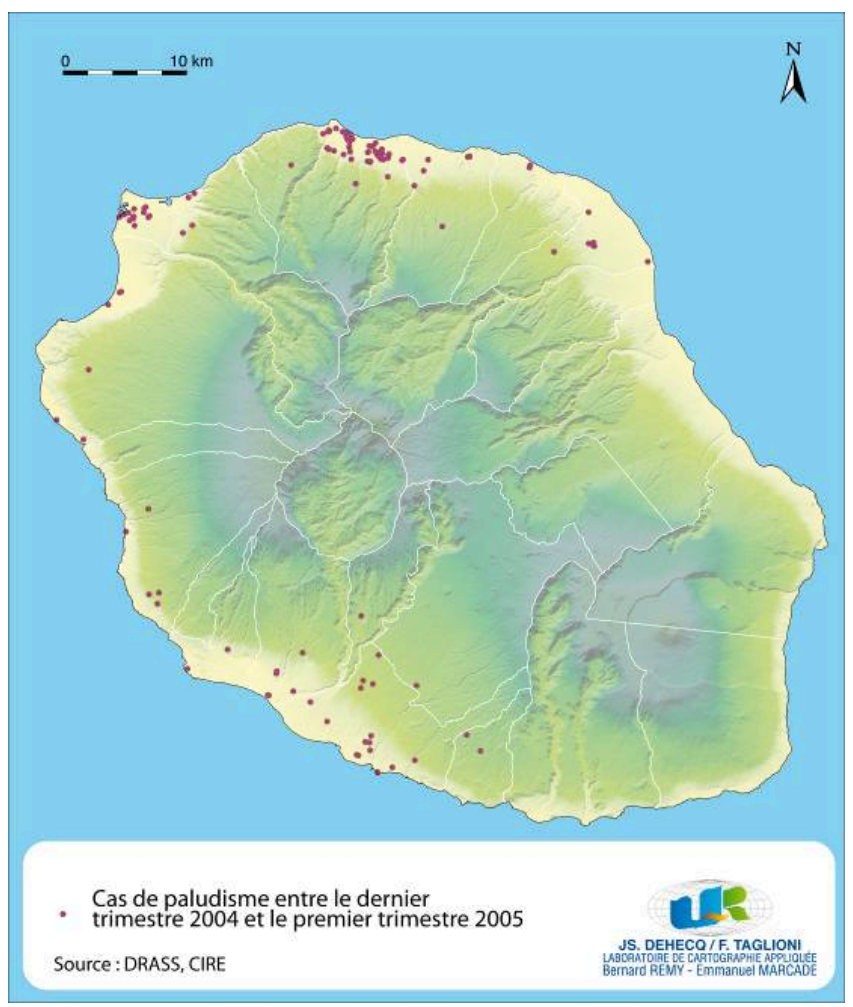

31 La première phase épidémique a présenté un pic en début mai 2005 avec 450 cas hebdomadaires (Renault, 2007). De janvier à août 2005, 2997 enquêtes géoréférencées ont été réalisées. Cette période présente l'avantage de patients contaminés par la 
souche virale de chikungunya sans la mutation. Cette mutation a été décrite à partir de novembre 2005 et confère au moustique une compétence vectorielle accrue à l'origine du second pic épidémique de grande ampleur (Vazeille, 2007 ; Tsetsarkin, 2007).

Pour conserver la même écologie du vecteur du chikungunya, Aedes albopictus, seules les zones urbaines situées à moins de 250 mètres d'altitude sont prises en compte. En effet, les données météorologiques de 4 stations du réseau Météo France situées sur 2 communes (St-Denis et St-Pierre) à 15 mètres et 250 mètres d'altitude à La Réunion montrent un écart maximal de $1,95^{\circ} \mathrm{C}$ entre les températures moyennes sur la période de mars 2005 à août 2005. Au-delà de cet écart, la dynamique de cette espèce pourrait être modifiée perturbant ainsi la transmission du virus chikungunya (Delatte, 2009). Nous ne retiendrons donc que les 2764 cas qui se trouvent en dessous de 250 mètres sur les 2997 cas géoréférencés.

Notre cadre d'étude des 2764 cas est donc ainsi défini :

- méthodologie constante d'enquête des cas de chikungunya (enquête individuelle de chaque signalement avec recherche active dans les 10 maisons voisines)

- homogénéité supposée de la souche virale qui, sans mutation, ne modifie pas la transmission par le vecteur sur la période d'étude

- écologie constante du vecteur à moins de 250m d'altitude

\subsection{Les foyers de cas de chikungunya}

34 La détermination des foyers de chikungunya retenus pour l'étude utilise la méthode d'analyse spatio-temporelle de Kulldorff $(1997 ; 1998)$ par le logiciel SATSCAN ${ }^{6}$ (Kulldorff, 2006) fondée sur l'étude de la dispersion dans le temps et dans l'espace des cas autour de chaque cas géoréférencé en dessous de 250 mètres. Un foyer est déterminé si un excès de cas est identifié à l'intérieur d'un agrégat temporel de cas par rapport au reste du territoire. Ainsi, seul l'intervalle de temps est choisi et le logiciel détermine la taille des foyers à partir de ces regroupements de cas dans le temps et l'espace. La taille du foyer est validée si la probabilité d'apparition d'un nouveau cas est plus importante dans le foyer qu'à l'extérieur par l'analyse de la dispersion de l'apparition de tous les cas sur le territoire. Cette méthode a été validée dans des études $\mathrm{du}$ lien spatio-temporel entre l'environnement et des foyers de maladies vectorielles ou non (Johnson, 2006, Hsu, 2007 ; Wagner, 2008).

Cette analyse statistique met évidence, sur un intervalle de temps de 7 jours entre les cas, 3 foyers épidémiques significatifs (fig. 9, fig. 10, fig. 11, fig. 12). Les autres intervalles de temps étudiés proposent des foyers regroupant plusieurs communes ne permettant pas une analyse fine. Cet intervalle de 7 jours rejoint les valeurs minimales de durée caractérisant le modèle de transmission du chikungunya : 2 jours de temps d'incubation extrinsèque chez le moustique et 5 jours d'incubation chez l'homme (Vazeilles, 2007; Nakoune, 2008). Les cartes des 3 foyers sont des représentations de l'ensemble des cas sur toute la période de l'étude de janvier à août 2005. Néanmoins, les agrégats de cas visibles hors des foyers sont apparus sur des périodes trop étendues pour être significatives par la méthode d'analyse Kulldorff utilisée. 
Figure 9. Distribution des 2997 cas de chikungunya à La Réunion déclarés de janvier à août 2005 et des 3 foyers identifiés

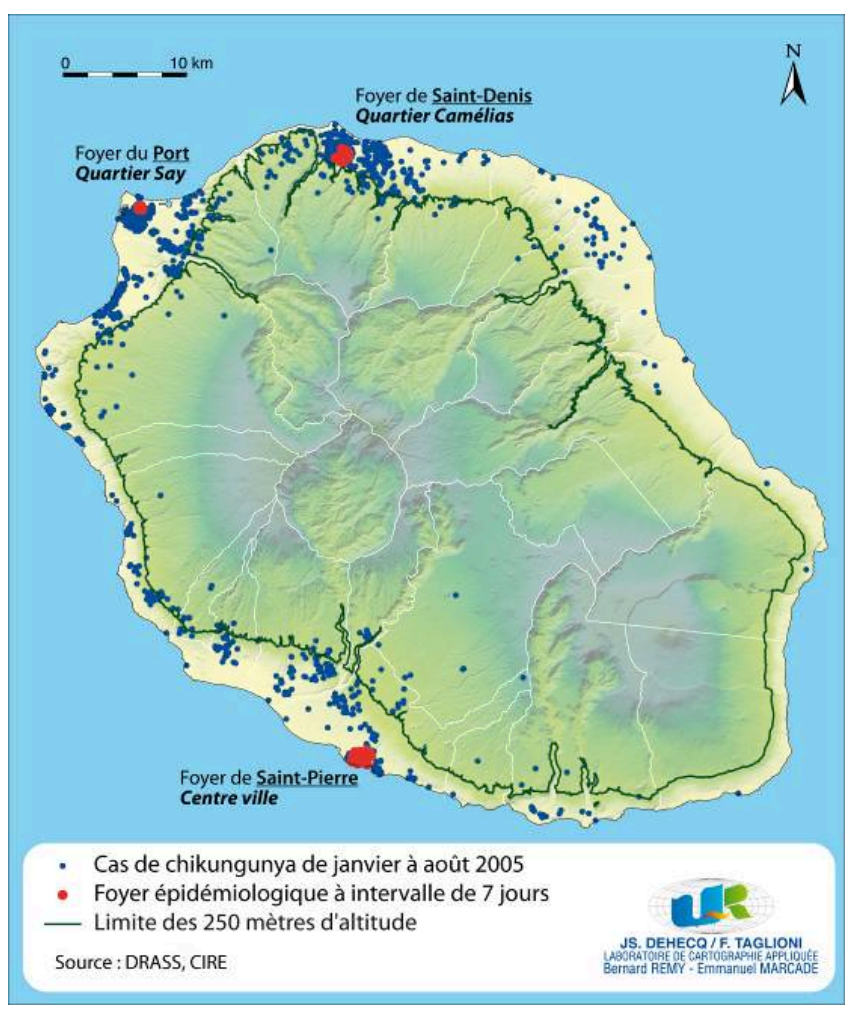

Figure 10. Foyer de cas de chikungunya du quartier Les Camélias à St-Denis

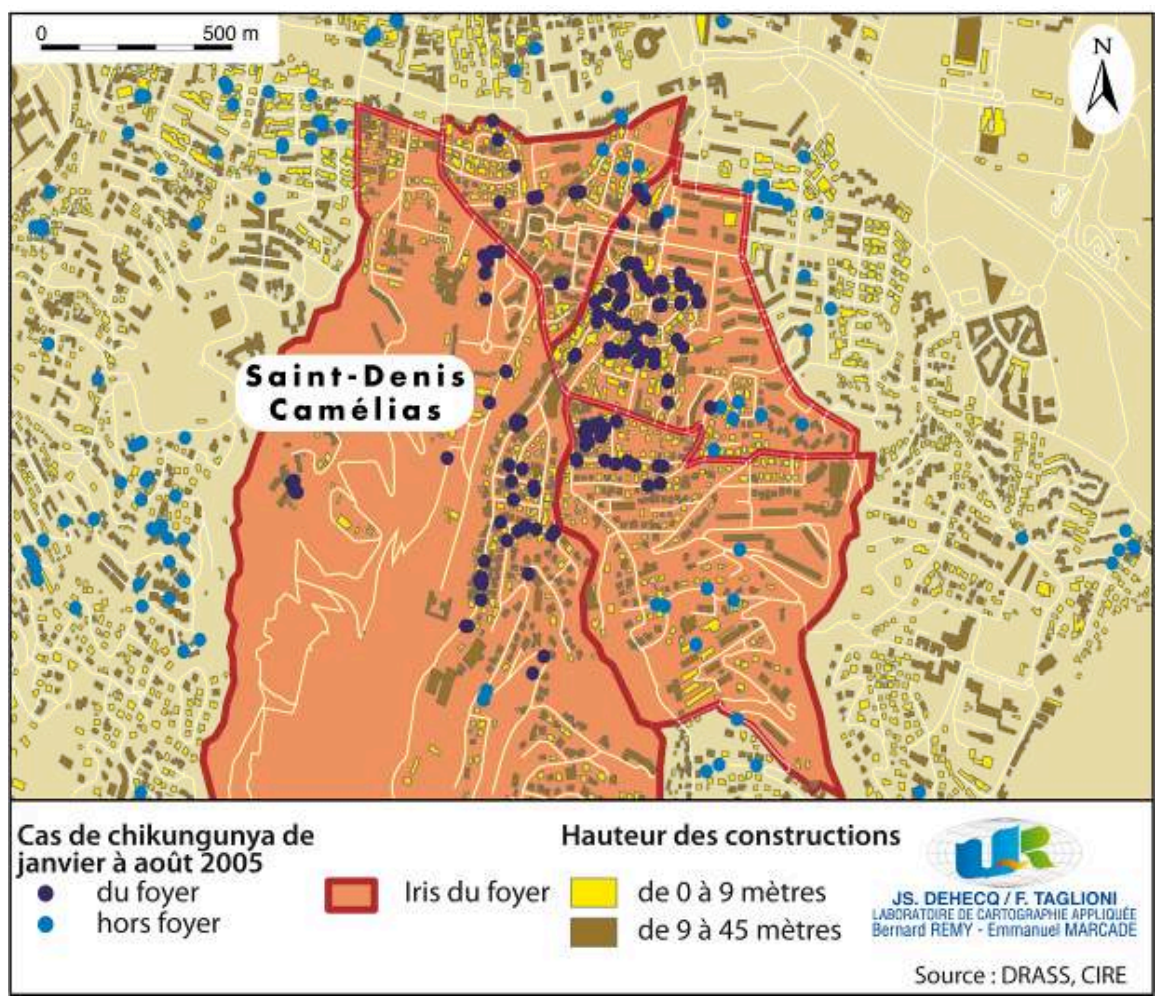


Figure 11. Foyer de cas de chikungunya du quartier Say à Le Port

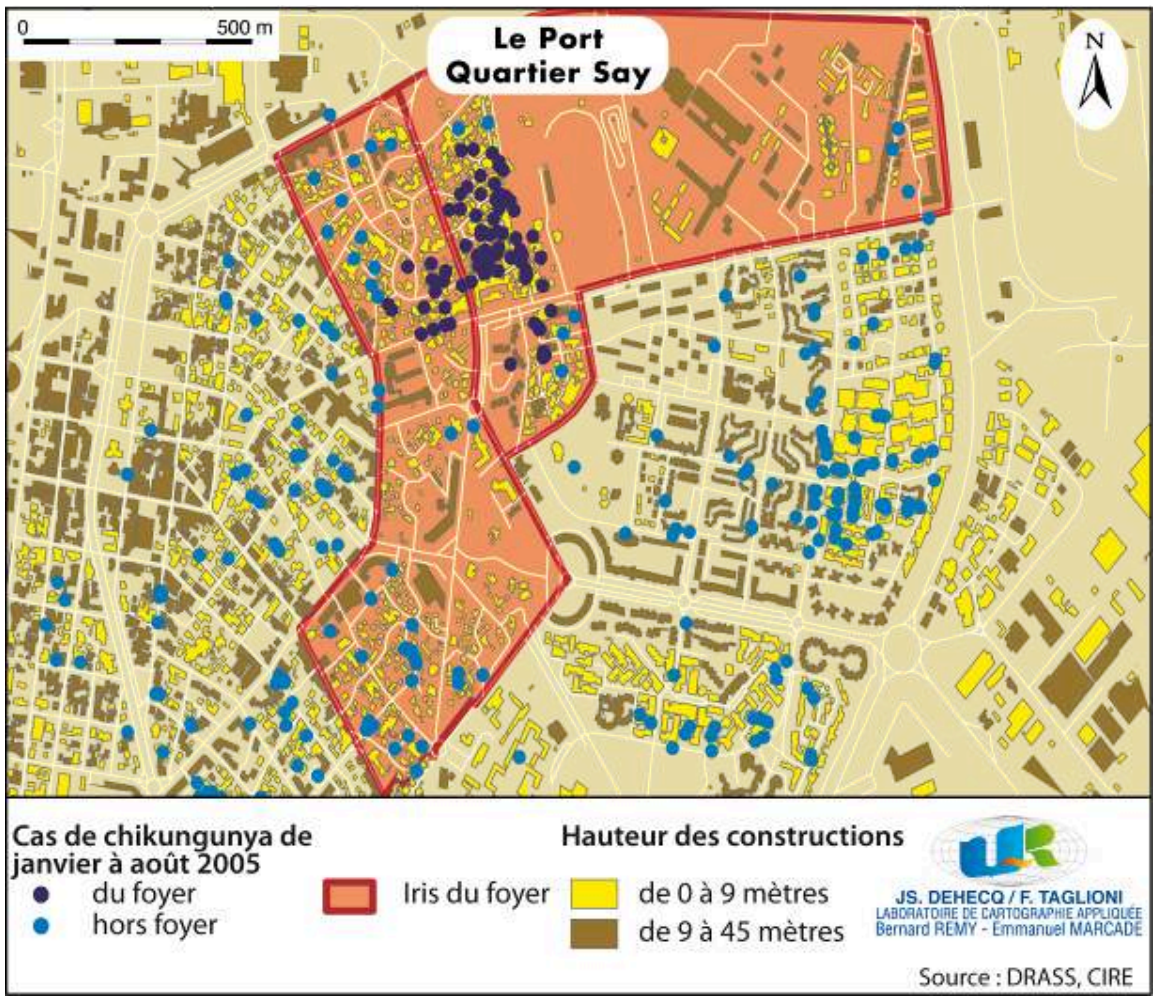

Figure 12. Foyer de cas de chikungunya du quartier Lavoir à St-Pierre

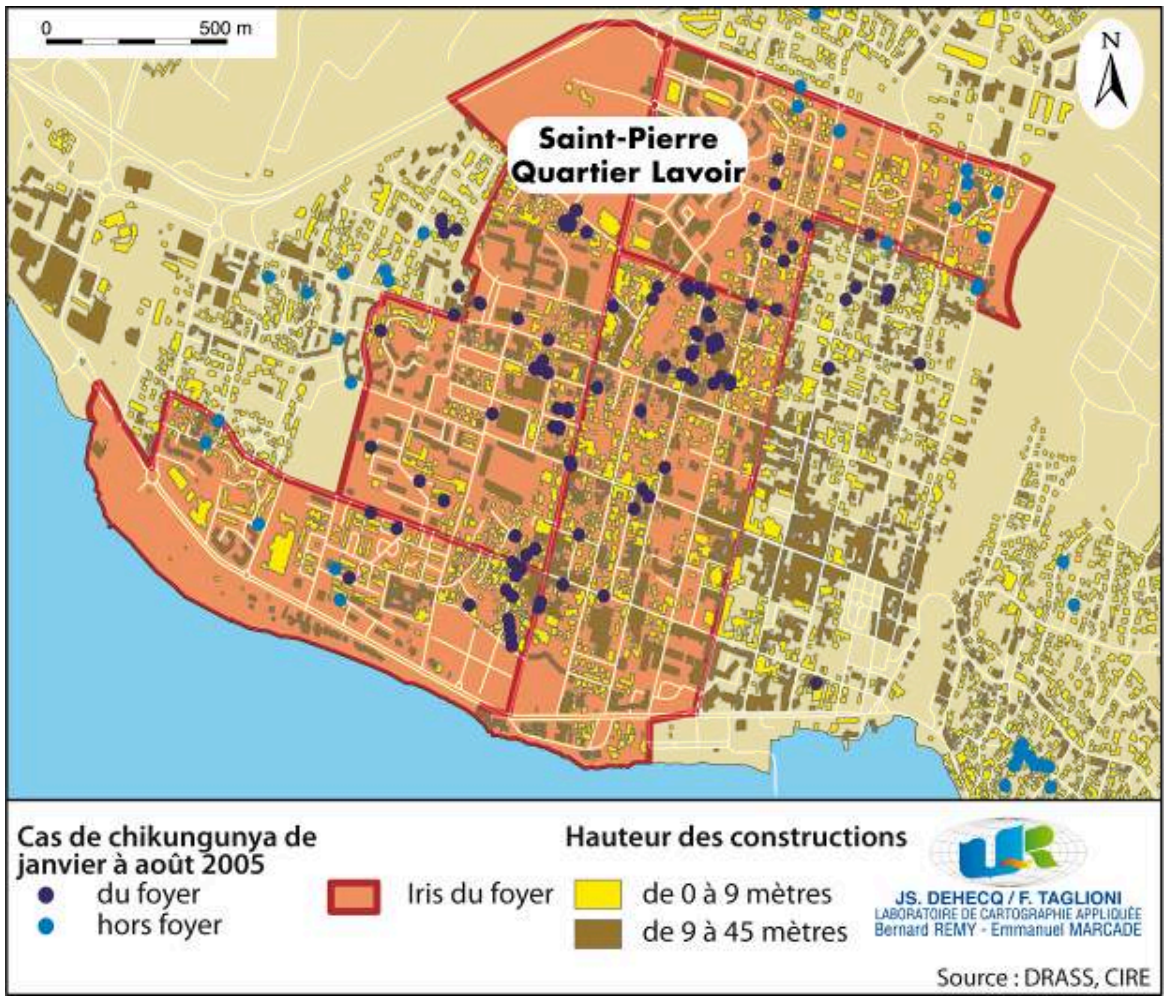


La distribution hebdomadaire des cas de chikungunya dans ces 3 foyers (fig. 13) se superpose parfaitement à la courbe épidémique globale pour La Réunion avec un pic des cas entre le 26 avril 2005 et le 10 mai 2005.

Figure 13. Distribution hebdomadaire des cas de chikungunya dans les 3 foyers de St-Denis Camélias, Le Port Say et St-Pierre Lavoir.

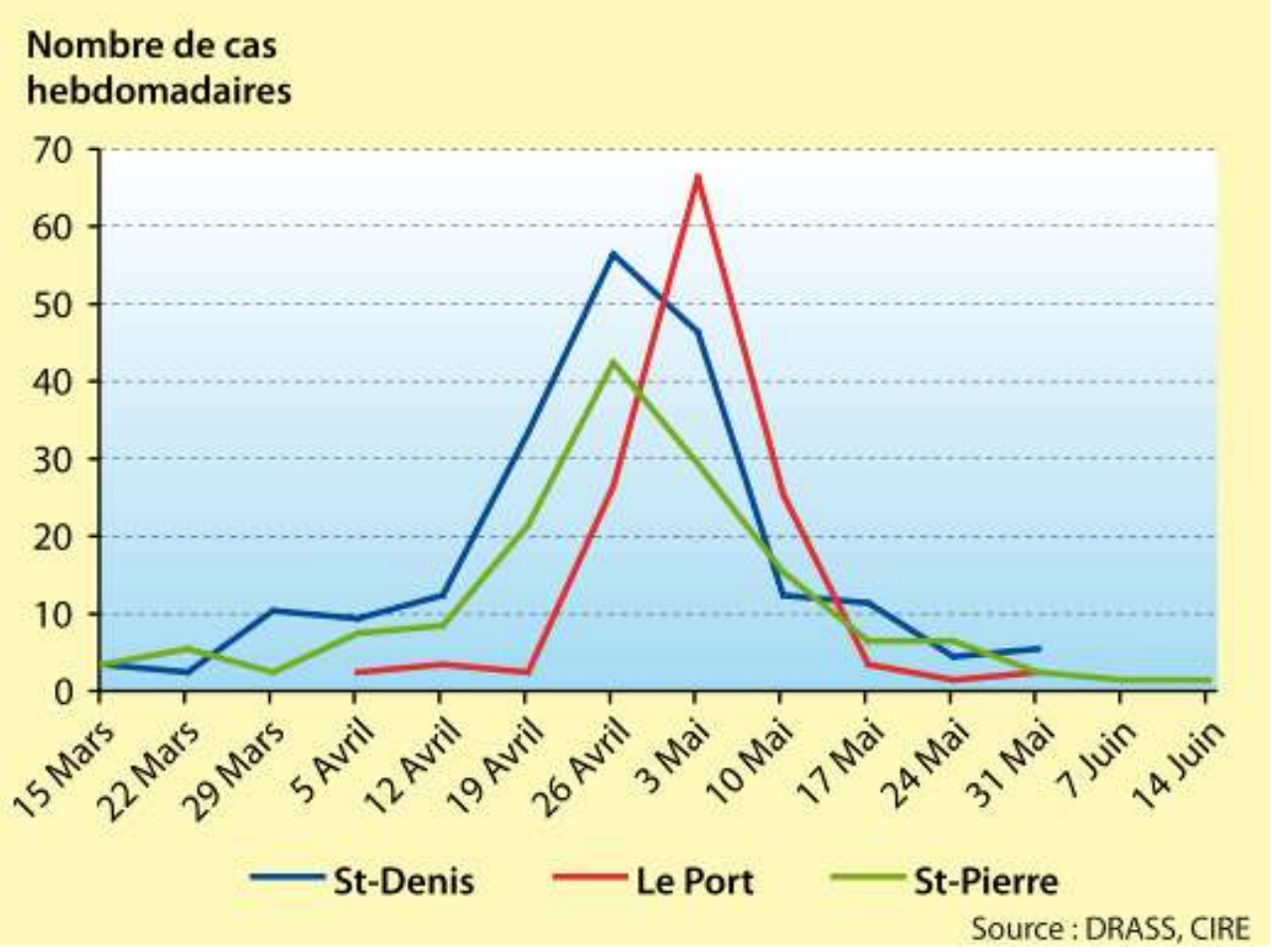

\subsection{Les données sociales et urbaines}

Les données relatives à la population et à l'habitat proviennent du dernier recensement général de 1999 de l'Institut national de la statistique et des études économiques (Inséé). Cette base de données apporte notamment des informations sur la structure démographique de la population, la structure des foyers, les niveaux de formation et activités professionnels et le logement de ces foyers. Les 573 variables proposées sont disponibles selon un découpage géographique de La Réunion en 344 Ilots regroupés pour l'information statistique (Iris) recouvrant toute l'île. Ce découpage géographique servira de référence afin de pouvoir lier les données sociales et urbaines à celles environnementales fournies par l'IGN. Pour illustrer ce découpage, précisons que les Iris, tels que définis par l'Inséé, sont constitués d'îlots eux aussi définis par l'Inséé ; c'est ce que nous voyons sur la photo qui suit (fig. 14). 
Figure 14. Exemple de découpage en Iris et îlots Inséé (le port de Saint-Pierre, La Réunion)

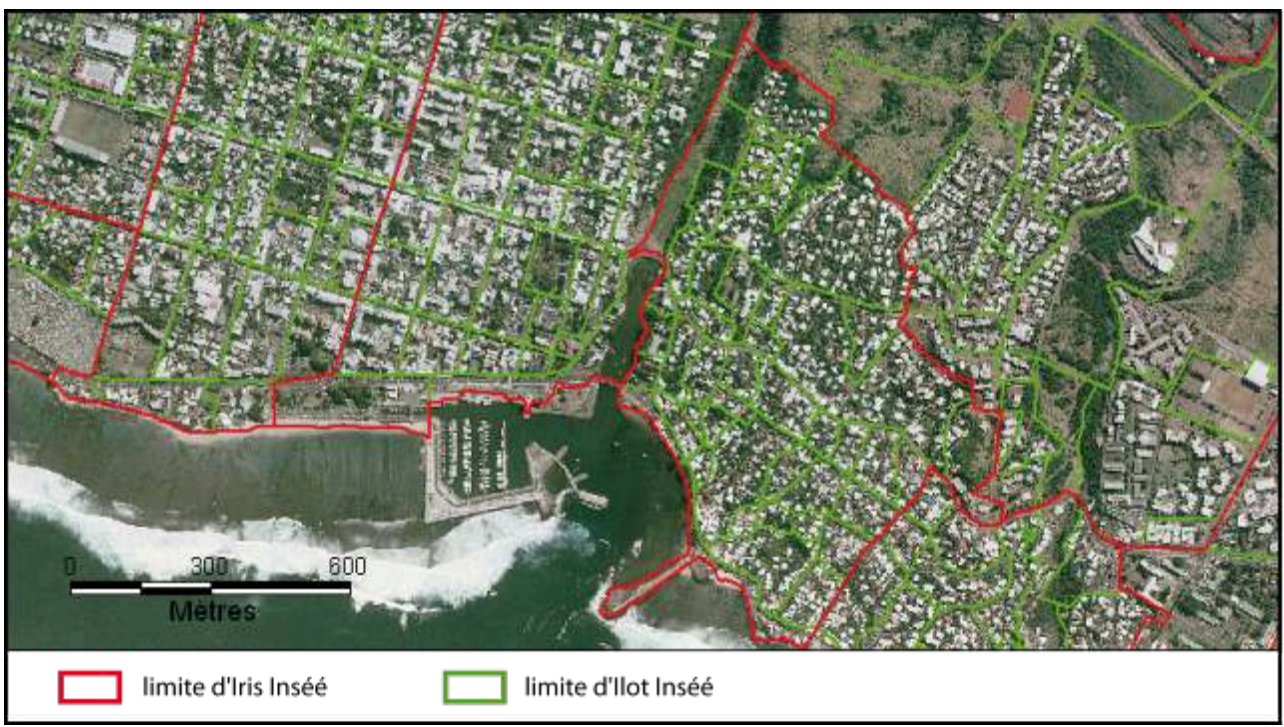

38 Le traitement des 573 variables a permis de générer 40 variables par regroupement et transformation de valeurs brutes en pourcentage décrivant :

- l'âge des populations

- l'activité professionnelle (chômage, temps partiel ou complet)

- les ressortissants étrangers

- le foyer : la taille, les habitants, l'âge et le type d'habitat (précaire, traditionnel ou récent)

- l'habitat collectif ou individuel

Les zones de découpage Inséé choisies pour les analyses sont celles qui superposent les 3 foyers. Ces 3 foyers rassemblent $18 \%$ des 2764 cas de la période d'étude. Ils chevauchent 12 Iris (sur les 175 couvrant l'île à moins de 250 mètres d'altitude) dont 10 sont retenus pour les analyses. En effet, 2 Iris ne présentent que quelques cas à la limite d'autres Iris retenus pour les foyers. Ces foyers, de superficie et de population variables, présentent des taux d'attaque apparents différents (fig. 15). Des données plus récentes et plus précises sont en cours de traitement par l'Inséé pour se superposer parfaitement à nos foyers d'étude. Elles permettront éventuellement d'affiner l'analyse le moment venu.

Figure 15. Données descriptives des 3 foyers identifiés

\begin{tabular}{|c|c|c|c|c|c|c|c|}
\hline $\begin{array}{l}\text { Nom } d u \\
\text { foyer }\end{array}$ & $\begin{array}{l}\mathrm{Nb} \text { de } \\
\mathrm{cas}^{\mathrm{a}}\end{array}$ & $\begin{array}{l}\text { Premiers et } \\
\text { derniers cas } \\
\text { du foyer }^{\mathrm{a}}\end{array}$ & $\begin{array}{l}\mathrm{Nb} \text { de } \\
\text { bâtis }\end{array}$ & $\begin{array}{l}\text { Superficie } \\
\text { (ha) }{ }^{b}\end{array}$ & $\begin{array}{l}\mathrm{Nb} \\
\text { d'habitants }\end{array}$ & $\begin{array}{l}\text { Taux de } \\
\text { d'incidence } \\
\text { cumuléc }^{\text {c }}\end{array}$ & $\begin{array}{l}\mathrm{Nb} \\
\text { Iris } \\
\text { Inséé }\end{array}$ \\
\hline $\begin{array}{l}\text { Les } \\
\text { Camélias }\end{array}$ & 210 & $\begin{array}{l}\text { du 26/02/05 } \\
\text { au 12/08/05 }\end{array}$ & 3656 & 47 & 9034 & $2 \%$ & 4 \\
\hline Say & 130 & $\begin{array}{l}\text { du 15/04/05 } \\
\text { au 07/06/05 }\end{array}$ & 1128 & 9 & 3488 & $4 \%$ & 2 \\
\hline
\end{tabular}




\begin{tabular}{|l|l|l|l|l|l|l|l|}
\hline Lavoir & 154 & $\begin{array}{l}\text { du 22/02/05 } \\
\text { au 17/07/05 }\end{array}$ & 3966 & 101 & 9806 & $2 \%$ & 4 \\
\hline
\end{tabular}

a. cas géoréférencés

b. surface du foyer de cas

c. nombre de cas de chikungunya du foyer rapportés à la population totale des Iris des foyers entre avril et août 2005

Les variables environnementales sont extraites de la base de données topographique (BD-Topo) datée de 2003 de l'Institut Géographique National (IGN). Elle décrit notamment l'occupation des sols en terme de morphologie urbaine (limites administratives, voies de circulation, aménagement structuraux, structure paysagère). Les variables retenues pour l'étude sont celles décrivant l'occupation des sols :

- bâti privé et public

- 2 variables ont été définies par les auteurs :

- taux de construction (pourcentage de la surface bâtie sur la surface constructible).

- taux d'habitat non collectif par hectare et par Iris et îlots inséé (pourcentage du bâti dont la hauteur n'excède pas 8 mètres).

\subsection{Etude des paramètres des foyers}

Chacune des 40 variables est testée statistiquement pour la caractérisation des 3 foyers à l'aide du test Mann et Whitney ${ }^{8}$ avec un seuil conservateur (p) de significativité inférieur ou égal à 0,1 pour tenir compte de la variabilité intrinsèque aux structures urbaines et les populations qui y vivent.

Les paramètres descriptifs significatifs associés aux 10 Iris de l'étude sont :

- la surface bâtie ( $\left.\mathrm{p}=1,310^{-3}\right)$ - fig. 16

- le pourcentage de logement à 1 ou 2 pièces $\left(p=3,410^{-3}\right)$ - fig. 17

- la densité de logements $\left(\mathrm{p}=4,910^{-3}\right)$ - fig. 18

- l'âge du bâti (construit avant 1981) ( $\left.\mathrm{p}=6,510^{-3}\right)$ - fig. 19

- le pourcentage de maisons individuelles sans étage $\left(\mathrm{p}=9,910^{-3}\right)$ - fig. 120

Les figures 16 à 20 représentent la moyenne et l'intervalle de confiance à $95 \%$ de ces 5 variables significatives des 10 Iris des foyers et de 165 Iris de La Réunion à moins de 250 mètres d'altitude (175 Iris) 
Figure 16. Surface bâtie

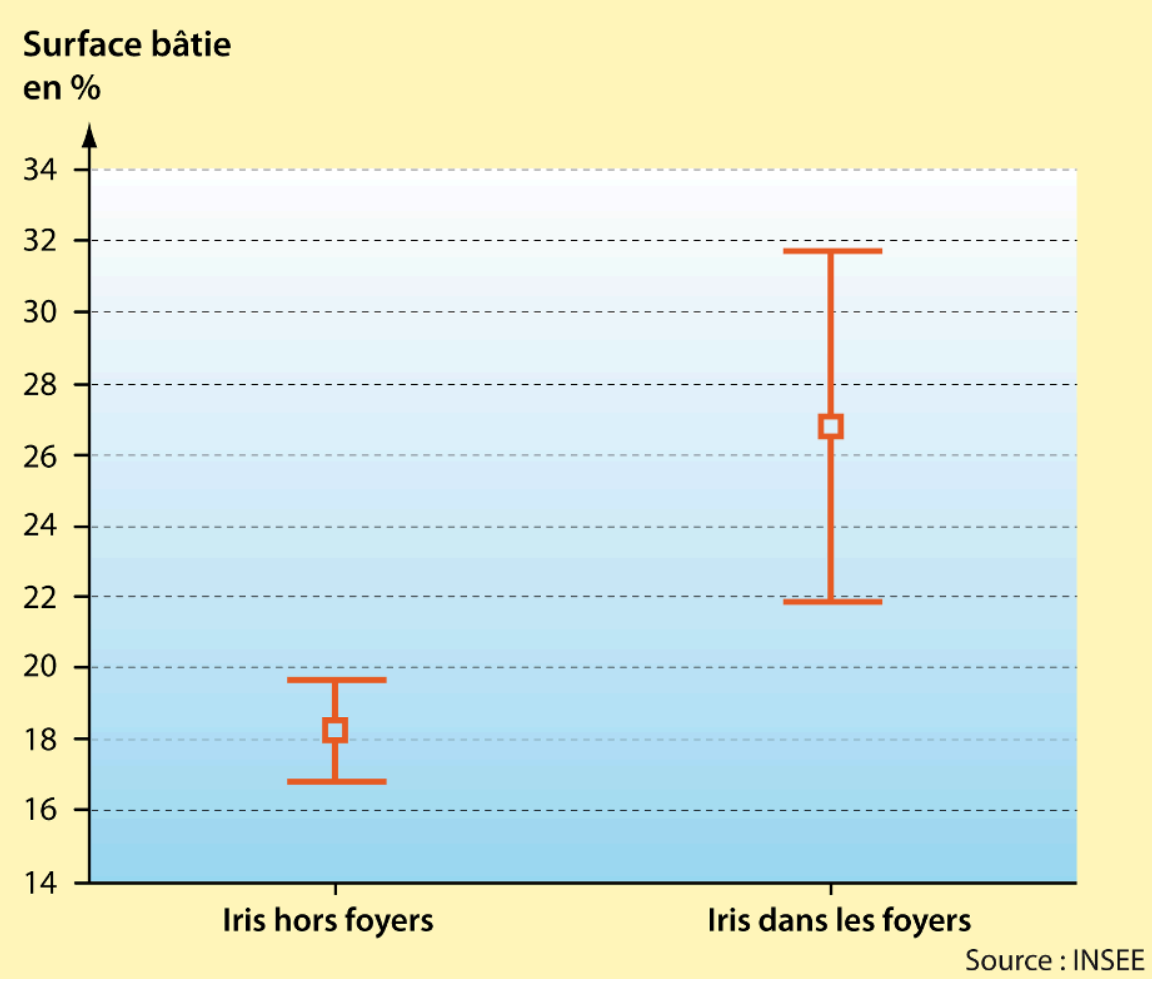

Figure 17. Pourcentage du logement avec 1 à 2 pièces

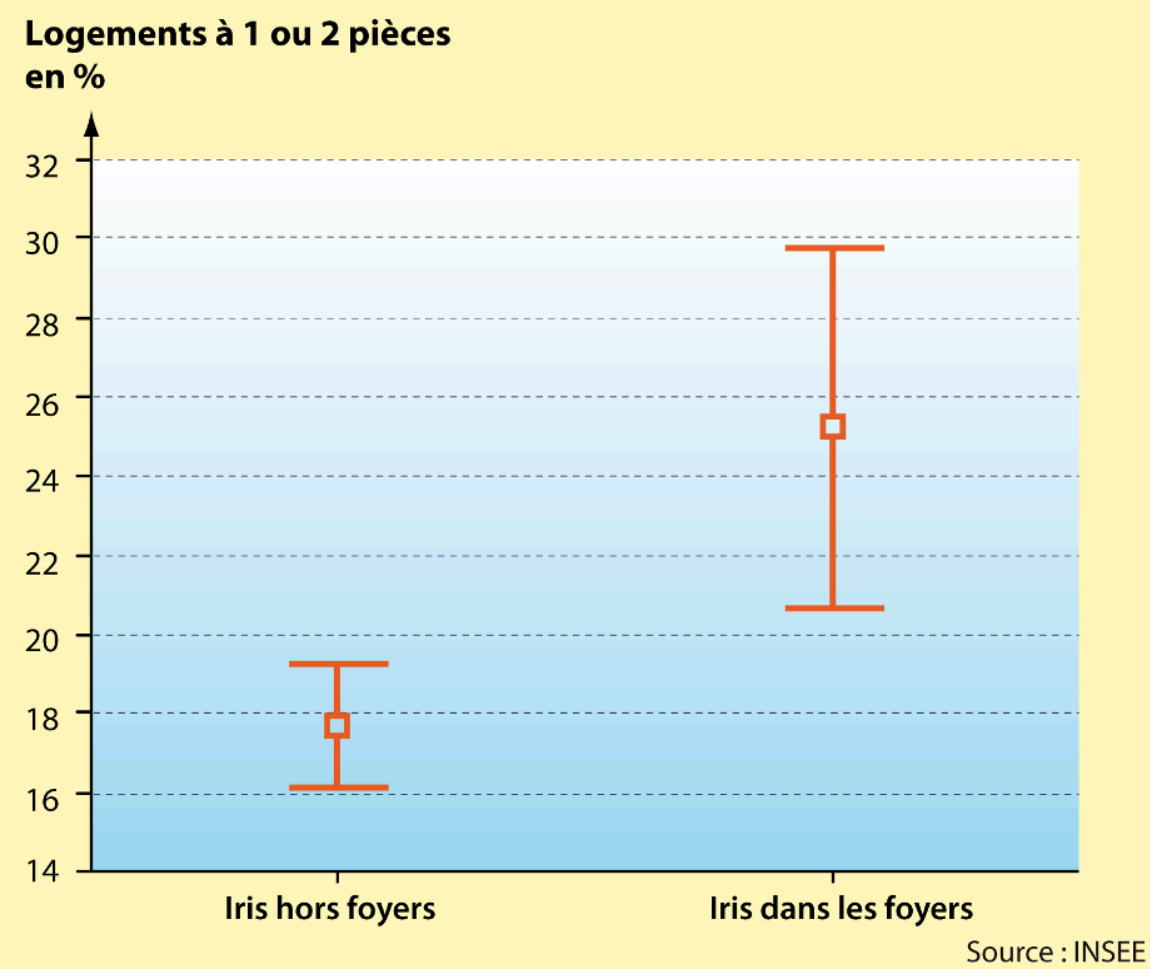


Figure 18. Densité du bâti

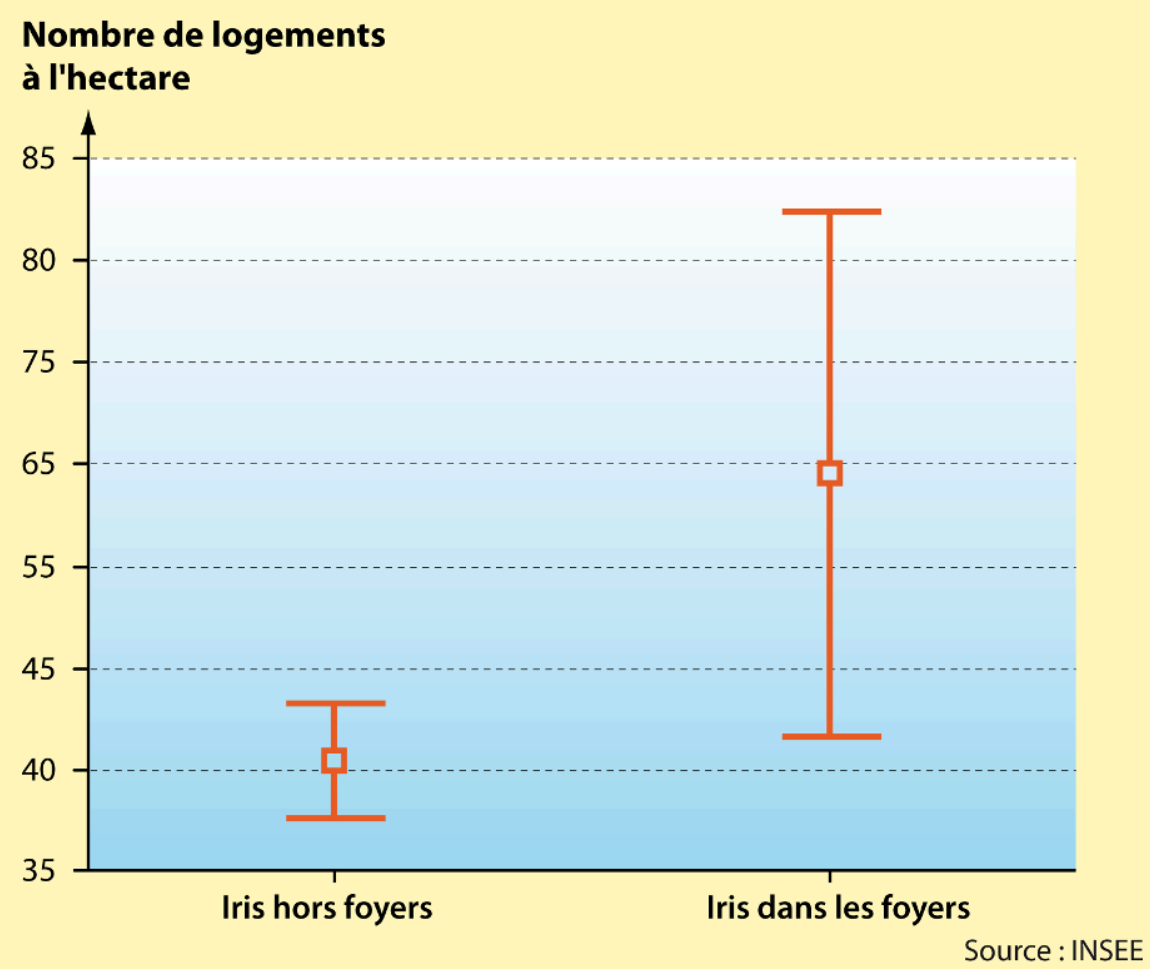

Figure 19. Pourcentage du bâti construit avant 1981

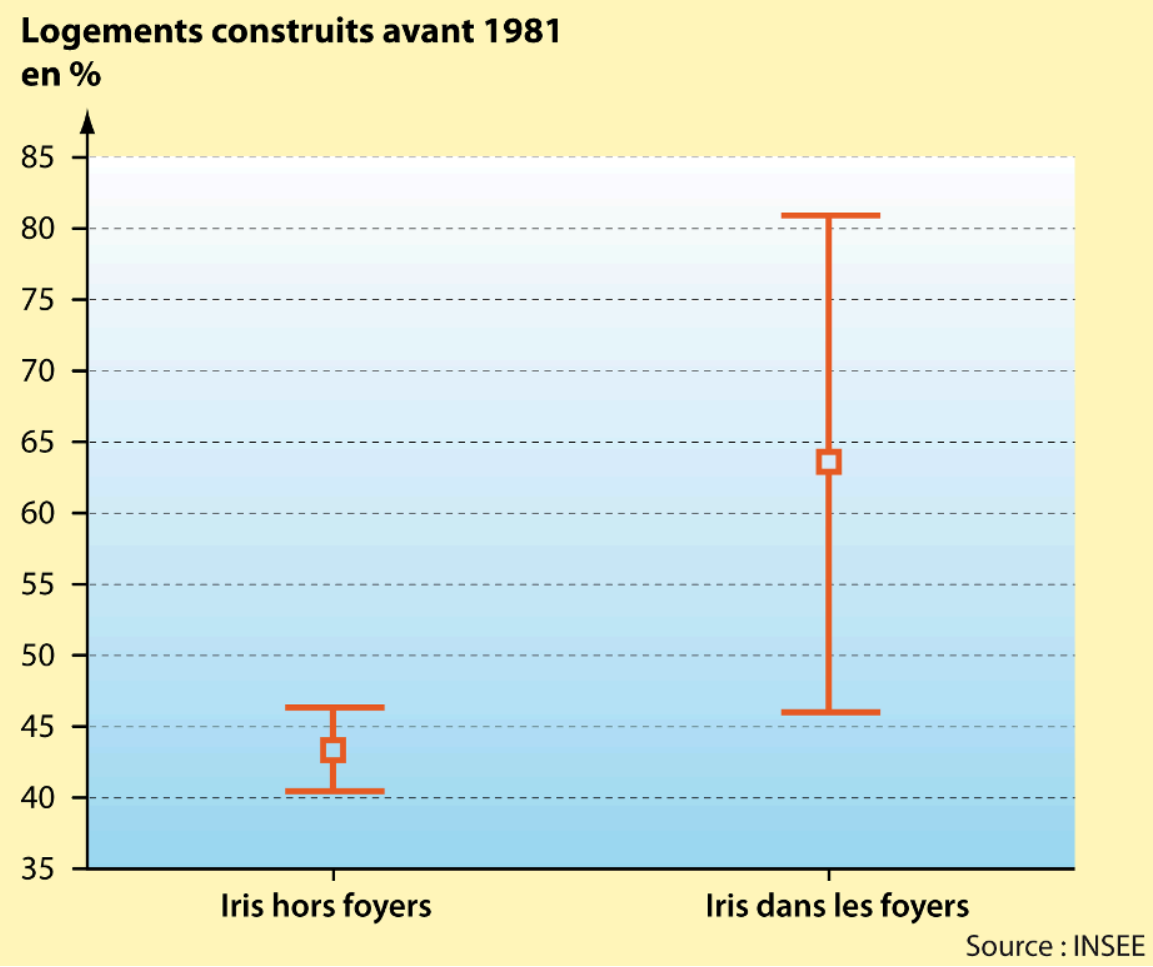


Figure 20. Pourcentage de maisons individuelles

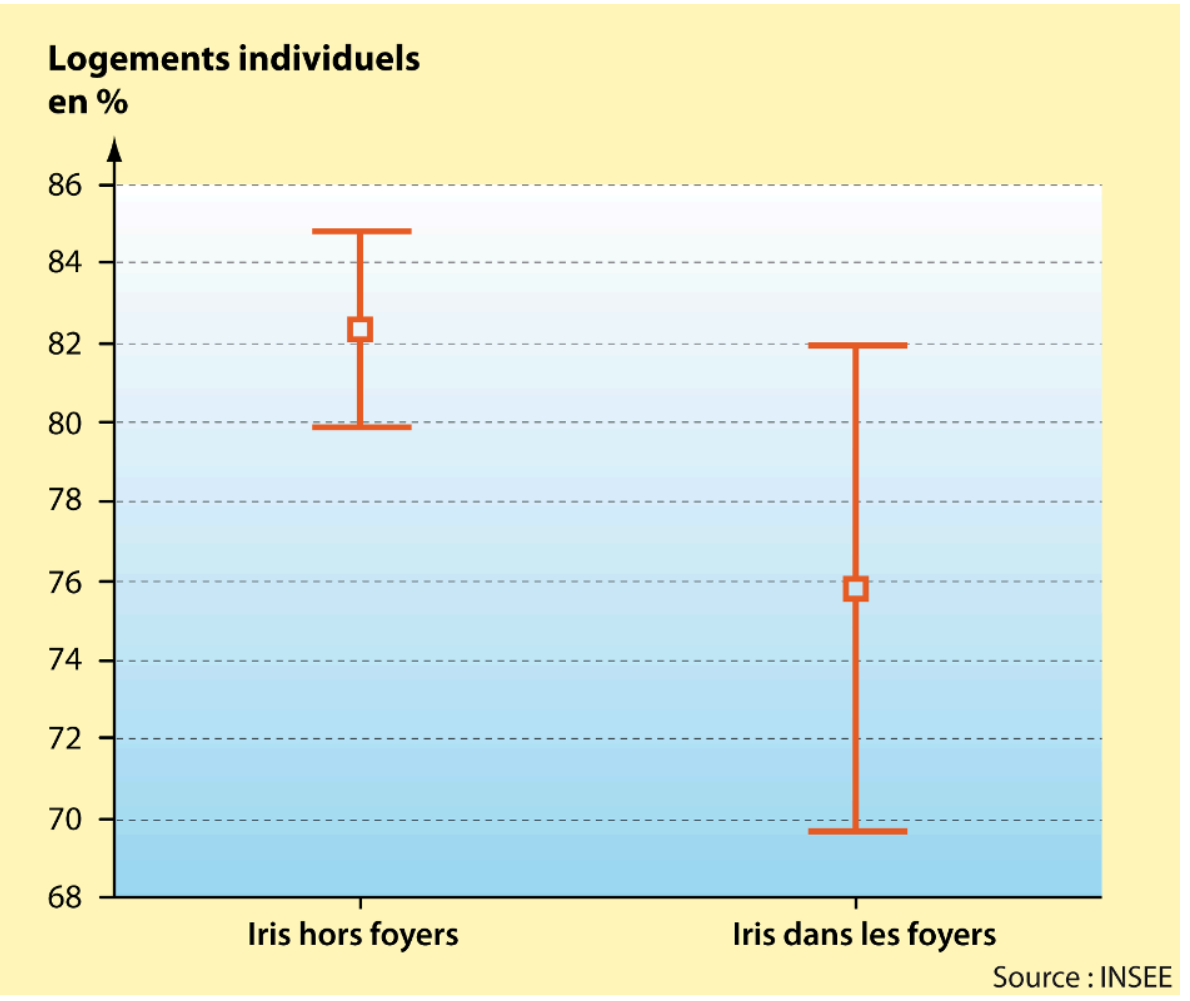

Les Iris comprenant des logements anciens, de petite taille et dont la densité du bâti est élevée sont ceux qui concentrent le mieux les premiers cas de chikungunya. Par ailleurs, l'analyse du pourcentage de maisons individuelles montre que les foyers sont ceux où la densité de logements individuels dans les Iris est inférieure à celle des autres Iris. Toutefois, une observation fine de ces Iris montre que les cas de chikungunya se distribuent au sein des zones d'habitats individuels denses. Par ailleurs, dans ces Iris on note que beaucoup de logements collectifs sont présents. Le découpage Iris ne permet pas d'isoler ce résultat observable sur les figures 10,11 et 12 où aucun cas n'est observé dans l'habitat collectif (plus de 8 mètres de haut) sur la période d'étude. Cette figure 20 illustre bien les contraintes d'échelle d'analyse mentionnées précédemment. Il y a une distorsion entre les Iris statistiques incontournables pour l'analyse et les réalités de terrain pour les dynamiques spatiales du chikungunya et de son vecteur.

On le constate, seuls des facteurs décrivant la morphologie urbaine ont été identifiés; les autres paramètres d'ordre social (âge, activité professionnelle ou niveau d'étude) évoqués au paragraphe 2.3 ne semblent pas significatifs ( $p>0,1)$. Néanmoins, ces facteurs sociaux sont pris en compte dans la mesure où ils sont liés à ces variables de morphologie urbaine (âge du logement, densité et surface construite). Ces liens peuvent être révélés par une analyse en composantes principales (ACP). Ces variables projetées sur le plan factoriel (fig. 21) montrent que la tranche d'âge de la population supérieure à 60 ans est corrélée à l'âge du bâti, que les variables locataires et habitat précaire (habitat traditionnel ou avec problème d'alimentation en eau et d'assainissement) sont liées à l'habitat individuel. Le facteur 1, caractérisé par l'habitat individuel dense (4 variables liées au logement individuel, à la densité du bâti, aux logements de 1 à 2 pièces et à la surface construite), est le plus explicatif de l'ACP. La forte inertie statistique $(61 \%)$ de ce facteur révèle une dichotomie entre habitat 
individuel dense et habitat collectif. Le facteur 2, dénommé âge du bâti, de portée explicative moindre, est caractérisé par cette seule variable.

Figure 21. Analyse en composantes principales (ACP) des variables significatives et de celles décrivant la population retenues dans l'étude

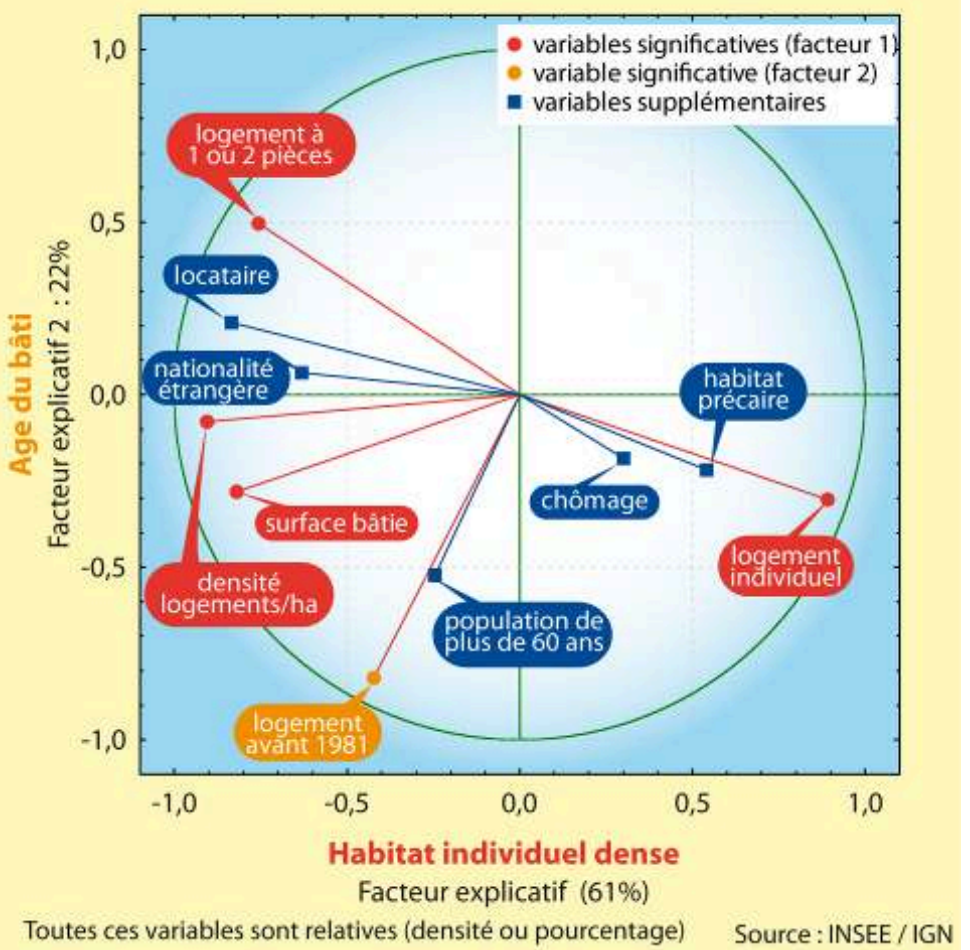

\subsection{Définition des quartiers à risques sanitaires}

Les paramètres significatifs retenus ci-dessus pour caractériser les 3 foyers permettent d'établir des valeurs seuils de zones à risques. Ainsi, un profil type de quartiers urbains à risques est défini pour l'ensemble de La Réunion à partir de ces données statistiques (fig. 22).

Figure 22. Tableau des valeurs seuils pour l'indentification des quartiers à risques

\begin{tabular}{|l|l|}
\hline & Iris Inséé \\
\hline Logements construits avant 1981 & $>48 \%$ \\
\hline Logements avec 1 à 2 pièces & $>20 \%$ \\
\hline Densité de logements (logements/ha) & $>16$ \\
\hline Surfaces construites & $>14 \%$ \\
\hline
\end{tabular}



de l'île dans la zone d'étude (moins de 250 mètres d'altitude) sont concernées. Il apparaît que 4 communes sont plus exposées que les 6 autres : St-André '(4 Iris), StDenis (26 Iris), St-Pierre (7 Iris) et Le Port (11 Iris). Au total, ces quartiers à risques se répartissent sur 63 Iris sur les 175 présents à moins de 250 mètres d'altitude. Ils sont, sans surprise, tous dans des zones de concentration urbaine et donc de concentration de population ainsi que des zones propices au vecteur Aedes alpobictus.

Figure 23. Carte des quartiers à risques sanitaires situés à moins de 250 mètres d'altitude

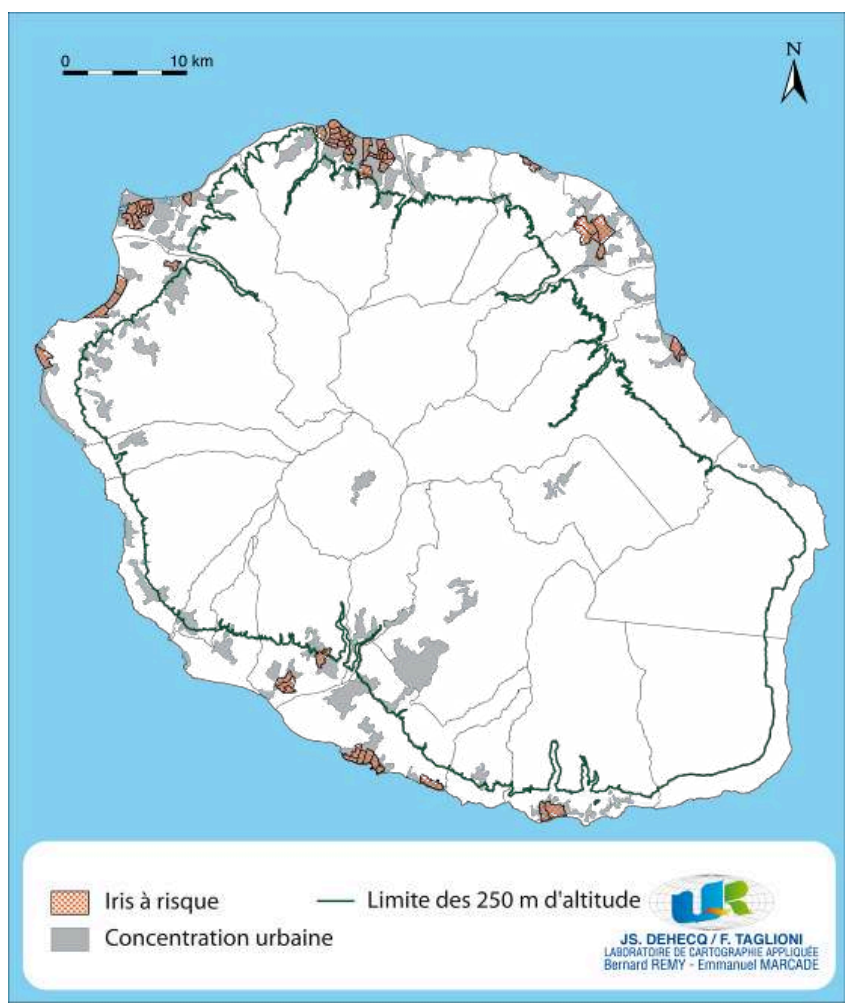

Cette deuxième partie de l'article démontre la présence de caractères significatifs communs à tous les premiers foyers de chikungunya à La Réunion. Ces facteurs d'exposition au risque de transmission d'arboviroses se rapportent majoritairement à la morphologie urbaine et donc indirectement à certaines variables sociales. En effet, si aucune variable descriptive des populations n'est significative, en revanche certaines semblent corrélées à des variables de morphologie urbaine. Même si ce lien entre l'habitat et certaines populations existe, l'hétérogénéité de l'habitat à l'intérieur des Iris perturbe cette mise en évidence.

L'extension de ces paramètres descriptifs à l'ensemble des Iris a permis de définir une carte des zones à risques de transmission d'arboviroses par Aedes albopictus. Cette carte montre que $36 \%$ des Iris situés à moins de $250 \mathrm{~m}$ d'altitude sont à risques. Les 4 communes présentant le plus grand nombre d'Iris à risques (St-Denis, Le Port, St-André et St-Pierre) rassemblent $41 \%$ de la population totale réunionnaise. Ce résultat 
démontre la nécessité d'une stratégie de gestion de ce risque sur ces communes réparties sur toutes les côtes de l'île (Nord, Est, Sud et Ouest).

L'échelle géographique de l'étude (Iris) permet de démontrer que certaines morphologies urbaines sont caractéristiques d'un risque d'exposition aux arboviroses pour les populations y résidant. Néanmoins, cette échelle masque l'effet d'autres variables socio-économiques sans doute pertinentes. D'autre part, il est probable qu'une certaine mixité sociale à La Réunion atténue d'autant l'effet potentiel de ces variables socio-économiques.

Au-delà de la seule observation des densités vectorielles (mesure de l'indice de Breteau'), la prise en compte de l'environnement socio-spatial est donc primordiale pour caractériser le risque d'exposition aux arboviroses. D'autres études sur des épidémies du virus de la maladie west-nile (Ruiz, 2004), de dengue (Van Benthem, 2005 ; de Mattos Almeida, 2007) ou de maladies non vectorielles (Linard, 2007 ; Chan-yeung, 2005) démontrent la nécessité de suivre tant le vecteur que son environnement urbain pour caractériser le risque et mettre en place des stratégies de gestion et des recommandations.

La présente analyse pourrait être affinée par une étude fine des 3 foyers à une autre échelle spatiale. Ainsi, la subdivision des 345 Iris en 9769 îlots pourrait servir d'unité géographique de référence à condition d'obtenir des données adaptées à cette échelle. Dans cette perspective, des enquêtes de terrain vont être menées dans les îlots des foyers et dans ceux voisins répondant aux critères à risques mais sans cas observé sur la période. Ces enquêtes auront pour objectifs :

- de préciser l'habitat et le milieu de chaque foyer pour décrire le jardin et le type de logement,

- d'enquêter les personnes du foyer pour décrire la structure du foyer, le nombre de personnes ayant eu le chikungunya et définir certains comportements de gestion de leur environnement proche,

- de décrire le quartier pour la typologie des voies de circulation et la présence de terrains et maisons abandonnées, de dépôts sauvages de déchets, de terrains publics et de canaux ou rivières,

- de préciser le lien entre l'environnement urbain et les populations y résidant.

\section{Conclusion}

A l'échelle régionale, l'épidémie de chikungunya a amplifié la prise de conscience des autorités nationales, régionales et internationales de la nécessité de mettre en œuvre des compétences, des réseaux, des ressources humaines et financières pour lutter contre les maladies émergentes. Un Réseau de surveillance et d'investigation des épidémies (RSIE) a été lancé en 2009 entre les membres de la Commission de l'océan Indien $\left(\mathrm{COI}^{10}\right)$. Ce réseau, sans préjuger de son efficacité, est un signe fort qu'en matière de santé mieux vaut parier sur les partenariats et la bonne volonté des Etats que sur la contrainte juridique du nouveau règlement sanitaire international qui reste dans les faits difficile à appliquer dans les pays les moins développés et les plus instables politiquement comme Madagascar par exemple. Un autre exemple fort de cette préoccupation sanitaire régionale est la création par la France du Centre de recherche et de veille sur les maladies émergentes dans l'océan Indien (CRVOI) dont les locaux sont à La Réunion. Ce Groupement d'intérêt scientifique (GIS) a vu le jour en 2007 dans 
le contexte de l'épidémie de chikungunya. Le CRVOI est apparu dans un premier temps comme une simple réponse politique de l'état à une crise qu'elle avait eu du mal à gérer. Après deux ans de fonctionnement, le CRVOI est devenu une référence dans le sud-ouest de l'océan Indien et au-delà en matière de recherche sur les maladies infectieuses émergentes.

56 L'une des grandes difficultés pour faire avancer les connaissances des facteurs systémiques d'émergence des maladies infectieuses à transmission vectorielle à l'échelle régionale est la multiplicité des variables à prendre en compte, le peu de fiabilité des sources de données et des données elles-mêmes, les grandes disparités de développement entre les Etats et territoires ainsi que les rivalités, voire conflits, issues de l'histoire et de la géographie, politiques et économiques, entre ces pays. A cela se superposent des enjeux de politiques nationales, notamment économiques, qui rendent difficiles à cerner les contours de l'environnement socio-spatial des épidémies ainsi que la mise en place d'une véritable politique de coopération régionale en matière sanitaire qui dépasse les clivages et les intérêts nationaux. Néanmoins, certaines variables comme le niveau de développement économique, le taux d'urbanisation, le degré d'ouverture des territoires au monde et les flux humains qui leur sont associés, les politiques nationales en matière de prévention ou encore le poids et l'influence médiatique sont autant de facteurs, qui dans le cas de l'épidémie de chikungunya, nous éclairent sur les interactions entre environnement et santé.

A l'échelle de La Réunion, on peut affirmer que l'exposition des personnes aux piqûres en milieu urbain est dépendante de leur mode de vie et de la structure urbaine des villes. Au-delà de l'aspect aléatoire de l'introduction de ce virus sur l'île, ces facteurs jouent un rôle majeur dans l'émergence de premiers foyers. Ces premiers foyers de chikungunya observés dés avril 2005 révèlent les zones les plus propices au développement de cycles de transmission locale. Les premiers résultats fondamentaux pour la mise en place d'une stratégie de gestion du risque de la population aux maladies vectorielles se dessinent. Ainsi, des actions de prévention pourraient s'orienter autour des quartiers à risques (fig. 22) que nous avons établi à partir d'une analyse épidémiologique exhaustive de près de 3000 cas géoréférencés durant la première phase de l'épidémie à La Réunion en 2005. Cette recherche action pourrait inspirer la mise en place de campagnes d'éducation sanitaire de proximité, de surveillance entomologique et de lutte préventive pour anticiper le risque sanitaire à transmission vectorielle sur l'île. Des études complémentaires en cours permettront d'affiner les résultats pour caractériser les facteurs, autres que ceux entomologiques, influençant la transmission et identifier les actions permettant de réduire ce risque d'exposition. Ce risque reste important dans le sud-ouest de l'océan Indien en général et à La Réunion en particulier avec une circulation permanente sur le mode épidémique, voire endémique à poussée épidémique, de maladies infectieuses comme le chikungunya, la dengue, la fièvre de la vallée du rift ou encore le paludisme.

Ce média ne peut être affiché ici. Veuillez vous reporter à l'édition en ligne http:// 


\section{BIBLIOGRAPHIE}

Anyamba, A. et alii, 2009. « Prediction of a Rift Valley fever outbreak ». Proceedings of the national academy of sciences of the united states of America, $\mathrm{n}^{\circ} 3$, p. 955-959

Ali, M. et alii, 2003. « Use of a geographic information system for defining spatial risk for dengue transmission in Bangladesh: role for Aedes albopictus in an urban outbreak ». The American journal of tropical medecine and hygiene, $n^{\circ} 6$, p. 634-640.

Aubry, P. ; Gaüzere, A., 2008. « Histoire des dengues et des syndromes dengue-like dans l'océan Indien». Bulletin de pathologie exotique, $n^{\circ}$ 101, p. 130

Bagny, L., et alii, 2009. «Aedes vectors of arboviruses in Mayotte : distribution area and larval habitats ». Journal of medical entomology, n², p. 198-207

Barbier, M., Prête, G., 2006. Un regard sociologique sur la biopolitique des maladies émergentes et réémergentes, Actes du colloque Emergences 2006, Paris, Octobre 2006, p. 130-139

Beck, U., 2001. La société du risque. Paris, Flamarion, 521 p.

Beesoon, S. et alii,, 2008. « Chikungunya Fever, Mauritius, 2006 ». Emerging Infectious Diseases, n², p. 337-338. http://www.cdc.gov/eid/content/14/2/pdfs/337.pdf

Benoist, J., 1987. « L'insularité comme dimension du fait social » in Îles tropicales : insularité, insularisme., 1987. Bordeaux, CRET, coll. "îles et archipels", nº, p. 37-44

Breiman, RF. et alii, 2008. «Lessons from the 2006-2007 Rift Valley fever outbreak in East Africa: implications for prevention of emerging infectious diseases ». Future virology, n5, p. 411-417

Brettes, A. et alii, 2001. « Le cholera à Mayotte ». Bulletin épidémiologique hebdomadaire, $\mathrm{n}^{\circ} 8$, Invs, p. 33-36

Carey, D., 1971. « Chikungunya and dengue : a case of mistaken identity? » Journal of the history of medecine and allied sciences, $n^{\circ} 3$, p. 243-262

Chan-yeung, M. et alii, 2005. «Socio-demographic and geographic indicators and distribution of tuberculosis in Hong Kong: a spatial analysis». International journal of tuberculosis and lung disease, $\mathrm{n}^{\circ} 12$, p. $1320-1326$

Chastel, C., 2006. Virus émergents, vers de nouvelles pandémies? Paris, Vuibert, 316 p.

Charrel, R. et alii, 2007. "Chikungunya outbreaks. The globalization of vector borne diseases », The New England Journal of Medecine, n8, p. 769-771

Collectif, 2008. « Qu'avons-nous appris de l'épidémie de chikungunya dans l'Océan Indien en 2005-2006?». Bulletin épidémiologique hebdomadaire (BEH), n³8-39-40, 21 octobre 2008, 44 p.

Collin, J; ; Paicheler, G., 2007. « Risque et santé », Sociologie et sociétés, n¹, p. 5-247

de Mattos Almeida, et alii, 2007. «Spatial vulnerability to dengue in a Brazilian urban area during a 7-year surveillance ». Journal of urban health, $n^{\circ} 3$, p. 334-345.

Coulanges, P., et alii, 1979. «Dengue à La Réunion. Isolement d'une souche à l'Institut Pasteur de Madagascar ». Bulletin de la société de pathologie exotique, $n^{\circ} 72$, p. 205-209.

Delatte, $\mathrm{H}$ et alii. 2009. « Influence of temperature on immature development, survival, longevity, fecundity, and gonotrophic cycles of Aedes albopictus, vector of chikungunya and dengue in the Indian Ocean ». Journal of medical entomology, $n^{\circ} 1$, p. 33-41 
Delatte, H. et alii., 2008a. « Aedes albopictus, vecteur des virus du Chikungunya et de la Dengue à la Réunion : biologie et contrôle ». Parasite, $n^{\circ} 15$, p. 3-13

Delatte, H. et alii., 2008b. « Geographic distribution and developmental sites of Aedes albopictus during a Chikungunya epidemic évent. Vector borne and zoonotic diseases, $\mathrm{n}^{\circ} 1$, p. 25-34

Fagherazzi-Pagel, H., 2007. Maladies émergentes et réémergentes chez l'homme. Paris, CNRS, 77 p.

Flahaut, A. ; Zyberman, P., 2008. Des épidémies et des hommes. Paris, Editions de la Martinière, 238 p.

Gonzalez, J-P. ; Saluzzo, J-F. ; Vidal, P., 2004. Les virus émergents. Paris, Ird Editions, 188 p.

Harpham, T. ; Stephens, C. 1991. «Urbanization and health in developing countries». World health statistics quarterly, $\mathrm{n}^{\circ} 2$. p. 62-69

Hsu, C. E. et alii, 2007. «A spatial-temporal approach to surveillance of prostate cancer disparities in population subgroups». Journal of the national médical association, $\mathrm{n}^{\circ} 1$, p. 72-80, 85-87

Idelson, B., 2009. « L'épidémie de Chikungunya à la Réunion. Médias, opinion publique et pouvoirs publics dans la crise ». Communication dans le colloque Chikungunya et autres arborviroses en milieu tropical, Saint-Pierre de la Réunion, 3-4 décembre 2007 (à paraître)

Invs, 2007. « Cas de chikungunya importés en Métropole durant l'épidémie à la Réunion Bilan de la surveillance à partir des données de laboratoire. $1^{\mathrm{er}}$ Avril $2005-31$ janvier 2007 ». Point au 22 mars 2007, 7 p.

http://www.invs.sante.fr/presse/2007/le_point_sur/chikungunya_cas_importes_230307/ chikungunya_cas_importes_230307.pdf

Johnson, G. et alii, 2006. « Geographic prediction of human onset of West Nile virus using dead crow clusters: an evaluation of year 2002 data in New York State ». American journal of epidemiology, $\mathrm{n}^{\circ} 2$, p. 171-180

Jones, K., 2008. « Global trends in emerging infectious diseases ». Nature, n 451, p. 990-993

Kles, V. et alii, 1994. « Enquêtes sérologiques concernant les arboviroses à Flaviviridae sur l'île de la Réunion ». Bulletin de pathologie exotique, $\mathrm{n}^{\circ}$ 87, p. 71-76

Knudsen, A. B. ; Slooff, R. 1992. « Vector-borne disease problems in rapid urbanization: new approaches to vector control ». Bulletin of the world health organisation, $\mathrm{n}^{\circ} 1$. p. 1-6

Kulldorff, M. et alii, 2006. «An elliptic spatial scan statistic ». Statistics in medecine, n²2, p. 3929-3943

Kulldorff, M. et alii, 1998. « Evaluating cluster alarms: a space-time scan statistic and brain cancer in Los Alamos, New Mexico ». Americanjournal of public health, n9, p. 1377-1380

Kulldorff, M. 1997. « A spatial scan statistic ». Communications in Statistics: Theory and Methods, $\mathrm{n}^{\circ} 26$, p. 1481-1496.

Linard, C. et alii , 2007. «Environmental conditions and Puumala virus transmission in Belgium ». International journal of health geographic, ${ }^{\circ} 6$, p. 55

Metselaar, D. et alii, 1980. « An outbreak of type 2 dengue fever in the Seychelles, probably transmitted by Aedes albopictus ». Bulletin de l'OMS, n 58, p. 937-943.

Mir, L., 2008. « Epidémie de chikungunya en Italie au cours de l'été 2007 ». Environnement, risques et santé, $n^{\circ} 3$, p. 163-164 
Morse, S., 2004. « Factors and determinants of disease emergence ». Revue scientifique et technique office international des épizooties, $\mathrm{n}^{\circ} 2$, p. 443-451

Mouchet, J. et alii, 1995. « La diffusion anthropique des arthropodes vecteurs de maladie dans le monde ». Cahiers santé, $\mathrm{n}^{\circ} 5$, p. 293-298

Mouchet, J.; Salvan, M., 1994. « Aedes Albopictus et Aedes Aegypti à l'île de La Réunion ». Annales de la société Belge de médecine tropicale, $\mathrm{n}^{\circ} 4,323-326$

Nakoune, E. et alii, 2007. « The Chikungunya virus ». Annale de biologie clinique, n 4 , p. 349-356

Onwujekwe, O. et alii, 2005. « Socio-economic inequity in demand for insecticide-treated nets, indoor residual house spraying, larviciding and fogging in Sudan ». Malaria journal, n4, p. 62.

Paquet, C. et alii, 2006. « Chikungunya outbreak in Reunion: epidemiology and surveillance, 2005 to early January $2006 »$. Eurosurveillance, $\mathrm{n}^{\circ} 2$

http://www.eurosurveillance.org/ViewArticle.aspx?ArticleId=2891

Ramchurn SK et alii, 2008. « Excess mortality as an epidemic intelligence tool in chikungunya mapping ». Eurosurveillance, $\mathrm{n}^{\circ} \mathrm{7}$ http://www.eurosurveillance.org/ViewArticle.aspx? ArticleId $=8039$

Raoult, D., 2005. Les nouveaux risques infectieux. Grippe aviaire, SRAS, et après? Paris, Lignes de repères, $272 \mathrm{p}$.

Ratsitorahina, M. et alii, 2008. " Outbreak of dengue and chikungunya fevers, Toamasina, Madagascar, $2006 »$. Emerging infectious diseases, ${ }^{\circ} 7$, p. 1135-1137

Renault, P. et alii, 2007. « A major epidemic of chikungunya virus infection on Reunion Island, France, 2005-2006 ». The American journal of tropical medecine and hygiene, n 4, p. 727-31

Rezza, G. et alii, 2007. «Infection with chikungunya virus in Italy : an outbreak in a temperate region ». Lancet, $\mathrm{n}^{\circ} 370$, p. 1840-1846

Ripert, C. (dir.), 2008. Epidémiologie des maladies parasitaires. Affections provoquées ou transmises par les arthropodes. Cachan, Editions médicales internationales, $580 \mathrm{p}$.

Ruiz, M. O. et alii, 2004. « Environmental and social determinants of human risk during a West Nile virus outbreak in the greater Chicago area, 2002 ». International journal of health geographic, $\mathrm{n}^{\circ}$ 1, p. 8

Salem, G., 1998. La santé dans la ville. Géographie d'un petit espace dense : Pikine (Sénégal). Paris, Karthala, 360 p.

Schwarz, T. et alii, 1994. « Hepatitis C and arboviral antibodies in the island populations of mauritius and Rodrigues ». Journal of medical virology, $\mathrm{n}^{\circ}$ 4, p. 379-383

Sergon, K. et alii, 2007. « Seroprevalence of chikungunya virus infection on Grande Comore island, Union of the Comoros ». American journal of tropical medecine and hygiene, n6, p. 1189-1193

Sissoko, D., et alii, 2009. « Rift valley fever, Mayotte-2007-2008 ». Emerging infectious diseases, $\mathrm{n}^{\circ} 4$, p. $568-570$

Sissoko, D., et alii., 2008. « Outbreak of Chikungunya fever in Mayotte, Comoros archipelagos, 2005 -2006 ». Transactions of the Royal Society of Tropical Medicine and Hygiene, n 8 , p. 780-786

Sissoko, D., et alii 2007. " Epidémie massive de fièvre chikungunya à Mayotte, France en 2005-2006 : description à partir des résultats de deux enquêtes épidémiologiques ». Invs, SaintMaurice, Bulletin épidémiologique hebdomadaire n48-49, p. 405-407 
Taglioni, F., 2009a. « Virus-sans-frontières : le chikungunya dans le sud-ouest de l'océan Indien ». Moullé,, F. (dir.), Frontières et santé. Genèses et maillages des réseaux transfrontaliers. Paris, L'Harmattan, p. 257-275. http://www.taglioni.net/Recherche/Sante.pdf

Taglioni, F., 2009b. « Le tourisme dans le sud-ouest de l'océan Indien : une durabilité à géométrie variable » in Vo Sang, XL (dir.) : Le tourisme durable, un instrument d'aide à la lutte contre la pauvreté ? Hanoi, Agence universitaire francophone (AUF)/Université Van Lang, p. 154-169 http://www.taglioni.net/Recherche/Vietnam.pdf

Taglioni, F., 2009c. «Chronique d'une crise sanitaire, économique et sociale. L'île de La Réunion face au Chikungunya ». Paris, UMR Prodig, Infogéo, 13 p. http://anastasie.univ-paris1.fr/infogeo/

Taglioni, F., 2008. «L'île d'Anjouan figure de la balkanisation de l'archipel des Comores ». EchoGéo, CNRS, rubrique Sur le vif" 2008, 12 p. http://echogeo.revues.org/index7223.html

Taglioni, F., 2005. « Les revendications séparatistes et autonomistes au sein des États et territoires mono- et multi-insulaires. Essai de typologie ». Cahiers de géographie du Québec, n¹36, p. 5-18 http://www.cgq.ulaval.ca/textes/vol_49/no136/03-Taglioni.pdf

Thammapalo, S. et alii, 2005. « Socio-demographic and environmental factors associated with Aedes breeding places in Phuket, Thailand ». Southeast Asian journal of tropical médecine and public health, $\mathrm{n}^{\circ} 2, \mathrm{p} .426-33$

Toma, B. ; Thiry, E., 2003. « Qu'est ce qu'une maladie émergente? ». Epidémiologie et santé animale, $\mathrm{n}^{\circ} 44$, p. $1-11$

Vallée, J., 2008. Urbanisation et santé à Vientiane. Thèse de doctorat de l'université Paris X, 354 p.

Van Benthem, B. H. et alii, 2005. «Spatial patterns of and risk factors for seropositivity for dengue infection ». The American journal of tropical medecine and hygiene $n^{\circ} 2$, p. 201-208

Vazeille, M. et alii, 2008. «Chikungunya : a risk for Mediterranean countries?». Acta tropica, $\mathrm{n}^{\circ} 2$, p. 200-202

Vazeille, M. et alii, 2007. « Two Chikungunya isolates from the outbreak of La Reunion (Indian Ocean) exhibit different patterns of infection in the mosquito, Aedes albopictus ». PLoS ONE, $n^{\circ} 11$. p. e1168

Wagner, T. et alii, 2008. « A Landscape-based model for predicting Mycobacterium ulcerans infection (Buruli Ulcer disease) presence in Benin, West Africa ». Ecohealth, n 1, p. 69-79

Watin, M., 2009. « Polémique, Rumeur et Tension : Aspects de la « crise » du chikungunya dans l'espace public médiatique réunionnais, in actes du colloque internationnal Epidémies et pharmacopee traditionnelle dans l'histoire des iles et pays de l'Océan indien occidental, Maurice, les 5, 6 et 7 décembre 2007 (à paraître)

Weinstein, P. ; Ravi, S., 2008. « The failure of colonial 'distancing': Changing representations of the 2005-06 chikungunya epidemic in Réunion, France ». Singapore Journal of Tropical Geography, $\mathrm{n}^{\circ} 29$, p. 221-235

Younus, M. et alii, 2007. « The role of neighborhood level socioeconomic characteristics in Salmonella infections in Michigan (1997-2007): assessment using geographic information system ». International journal of health geographic, $\mathrm{n}^{\circ} 6$, p. 56

Zeller, H., 1998. « Dengue, arboviruses and migrations in the Indian Ocean ». Bulletin de la société de pathologie exotique, $\mathrm{n}^{\circ} 1$, p. 56-60 


\section{NOTES}

1. Cette mesure relative qui rapporte un nombre de malades à la population totale est plus pertinente que le taux d'incidence cumulée qui ne prend en compte que les nouveaux cas sur une période rapportés à l'effectif des personnes non malades au début de la période. Le taux d'incidence est surtout utilisé pour les maladies chroniques.

2. Cela étant, en ce qui concerne les Seychelles ce taux officiel de $25 \%$ semble sous-estimé. D'après des informations de sources médicales locales fiables, bien que cela soit difficilement vérifiable en l'état, le taux de prévalence serait bien supérieur à $25 \%$.

3. Déclaration, le lundi 9 mars 2009 à Lyon de David Heymann, sous-directeur général de l'OMS pour la sécurité sanitaire et l'environnement, lors de la deuxième journée du Forum mondial des sciences de la vie, Biovision qui avait pour thème dominant la " gestion des épidémies urbaines ". 4. L'OMS estime que la dengue progresse territorialement et fait chaque année plus de 1200 morts.

5. http://air-seychelles.net/infos/chikungunya_moustique.html

6. version 7.0

7. Le taux apparent de prévalence a été calculé de la façon suivante: nombre de cas de chikungunya dans le foyer rapporté au nombre moyen de personne par habitations multiplié par le nombre de logements dans le foyer

8. Ce test permet la comparaison de 2 échantillons de taille différente d'une même population

9. L'indice de Breteau est égal au nombre de gîtes avec présence de larves dans 100 maisons enquêtées

10. La Commission de l'océan Indien, organisation intergouvernementale créée en 1984, compte 5 membres : Les Comores, Madagascar, Maurice, Les Seychelles, La Réunion. Ces actions portent sur 4 domaines en particulier: Coopération politique, diplomatique et de sécurité; Développement économique et insularité ; Ressources naturelles et environnement; Développement humain

\section{RÉSUMÉS}

Parmi le grand nombre possible d'aléas dans le sud-ouest de l'océan Indien (cyclones, pluies torrentielles, volcanisme, tsunami, maladies infectieuses ...) certains sont plus dévastateurs et présents que d'autres. L'épidémie de chikungunya, qui s'est déroulée de 2004 à 2006, est sans doute l'événement majeur de santé publique qui a marqué les hommes, les sociétés et les esprits aussi. Cette épidémie a touché les Etats et territoires de la région avec des prévalences de 25 à 60 $\%$. Pour essayer de caractériser les contours de la vulnérabilité et déterminer des effets de contexte nous avons mené une analyse des niveaux de développement humain, social et sanitaire des pays concernés. S'il est établi que les facteurs d'émergence à l'échelle régionale sont très largement liés à des variables socio-spatiales néanmoins, à aléa égal le risque n'est pas proportionnellement plus important dans les pays réputés les plus vulnérables. A l'échelle de La Réunion, une analyse des données de l'environnement socio-spatial et épidémiologique, avec près de 3000 cas géoréférencés durant la première phase de l'épidémie, permet d'établir les principaux quartiers à risques de l'île. 
Among the large amount of likely hazards in the South West of the Indian Ocean region (hurricanes, torrential rains, tsunamis, infectious diseases ...), some are more devastating and present than others. The Chikungunya epidemic that lasted from 2004 to 2006, as probably the major public health event, has left a strong impact on men, societies, and people's mind. This epidemic hit the states and territories in the region with $25 \%$ up to $60 \%$ prevalences. In an attempt to characterize the outlines of vulnerability and to determine contextual effects, we conducted an analysis of levels in human, social and health development in the involved countries. Although it has been established emergence factors on a regional scale are nevertheless widely related to social-spatial variables, and given equal hazard, the risk is not proportionally more important than in the known most vulnerable countries. On the scale of La Reunion, a data analysis of the social-spacial and epidemiologic environment with nearly 3000 cases georeferenced during the first phase of the epidemic, allows to assess the main areas at risk on the island.

\section{AUTEURS}

\section{FRANÇOIS TAGLIONI}

François Taglioni (tagli@diplomacy.edu) est directeur de recherche à l'IRD, UR 029, en affectation à La Réunion et membre de l'UMR PRODIG. Spécialiste des petits espaces insulaires en développement dans le monde, il travaille actuellement sur les interactions santé/ environnement dans le sud-ouest de l'océan Indien.

Site Internet de l'auteur : http://www.taglioni.net

\section{JEAN-SÉBASTIEN DEHECQ}

Jean-Sébastien Dehec (jsdehecq@ool.fr) est ingénieur entomologiste du service de Lutte antivectorielle (LAV) à la Direction régionale des affaires sanitaires et sociales (DRASS) à La réunion. Il a pour mission l'organisation et la programmation des suivis des activités, la définition des protocoles de lutte, la validation des techniques utilisées ainsi que la structuration du service et professionnalisation des agents du service. 